\title{
The bimodal colors of Centaurs and small Kuiper belt objects ${ }^{\star \star \star}$
}

\author{
N. Peixinho ${ }^{1,2}$, A. Delsanti ${ }^{3,4}$, A. Guilbert-Lepoutre ${ }^{5}$, R. Gafeira $^{1}$, and P. Lacerda ${ }^{6}$ \\ ${ }^{1}$ Center for Geophysics of the University of Coimbra, Av. Dr. Dias da Silva, 3000-134 Coimbra, Portugal \\ e-mail: [peixinho;gafeira] @mat.uc.pt \\ 2 Astronomical Observatory of the University of Coimbra, Almas de Freire, 3040-004 Coimbra, Portugal \\ ${ }^{3}$ Laboratoire d'Astrophysique de Marseille, Université d'Aix-Marseille, CNRS, 38 rue Frédéric Joliot-Curie, 13388 Marseille, \\ France \\ e-mail: Audrey.Delsanti@oamp.fr; Audrey.Delsanti@obspm.fr \\ ${ }^{4}$ Observatoire de Paris, Site de Meudon, 5 place Jules Janssen, 92190 Meudon, France \\ 5 UCLA, Department of Earth and Space Sciences, 595 Charles E. Young Drive East, Los Angeles CA 90095, USA \\ e-mail: aguilbert@ucla.edu \\ ${ }^{6}$ Queen's University Belfast, Astrophysics Research Centre, Belfast BT7 1NN, UK \\ e-mail: p.lacerda@qub.ac.uk
}

Received 16 February 2012 / Accepted 14 June 2012

\section{ABSTRACT}

\begin{abstract}
Ever since the very first photometric studies of Centaurs and Kuiper belt objects (KBOs) their visible color distribution has been controversial. This controversy has triggered to a prolific debate on the origin of the surface colors of these distant icy objects of the solar system. Two scenarios have been proposed to interpret and explain the large variability of colors, hence surface composition. Are the colors mainly primordial and directly related to the formation region, or are they the result of surface evolution processes? To date, no mechanism has been found that successfully explains why Centaurs, which are escapees from the Kuiper belt, exhibit two distinct color groups, whereas KBOs do not. We readdress this issue using a carefully compiled set of $B-R$ colors and $H_{R}(\alpha)$ magnitudes (as proxy for size) for 253 objects, including data for 10 new small objects. We find that the bimodal color distribution of Centaurs is a size-related phenomenon, common to both Centaurs and small KBOs, i.e. independent of dynamical classification. Furthermore, we find that large KBOs also have a bimodal distribution of surface colors, albeit distinct from the small objects and strongly dependent on the "Haumea collisional family" objects. When plotted in $B-R, H_{R}(\alpha)$ space, the colors of Centaurs and KBOs display a peculiar $\mathcal{N}$ shape.
\end{abstract}

Key words. Kuiper belt: general

\section{Introduction}

Discovered just 20 years ago (Jewitt \& Luu 1993), the Kuiper belt holds a vast population of icy bodies orbiting the Sun beyond Neptune. Stored at very low temperatures $(\sim 30-50 \mathrm{~K})$, the Kuiper belt objects (KBOs) are expected to be well-preserved fossil remnants of the solar system formation. Presently, $\sim 1600$ KBOs have been identified and classified into several dynamical families (see Appendix A and Gladman et al. 2008, for a review). Kuiper belt objects, which dynamically evolve to become Jupiter family comets (JFCs), form a transient population, the Centaurs, with short-lived chaotic orbits between Jupiter and Neptune (Kowal et al. 1977; Fernandez 1980; Levison \& Duncan 1997).

Between 1998 and 2003, we witnessed a debate on the surface colors of KBOs and Centaurs. One team used very accurate surface colors and found that KBOs were separated into two distinct color groups (Tegler \& Romanishin 1998, 2000, 2003). Other teams did not find evidence of any color bimodality

\footnotetext{
* Table 3 and Appendix A are available in electronic form at http://www . aanda.org

$\star \star$ Table 3 is also available at the CDS via anonymous ftp to cdsarc.u-strasbg.fr $(130.79 .128 .5)$ or via

http://cdsarc.u-strasbg.fr/viz-bin/qcat?]/A+A/546/A86
}

(Barucci et al. 1999; Jewitt \& Luu 2001; Hainaut \& Delsanti 2002). Careful reanalysis of the data by Peixinho et al. (2003) indicated that only the Centaurs display bimodal colors, i.e. they are distributed into two distinct color groups, one with neutral solar-like colors and the other with very red colors. On the other hand, KBOs exhibit a broad continuous color distribution, from neutral to very red, with no statistical evidence of a color gap between the extrema (Tegler et al. 2008, for a review).

The relevance of this controversy lies in two possible interpretations: i) KBOs and Centaurs are composed of intrinsically different objects, with distinct compositions, which probably formed at different locations in the protosolar disk; ii) KBOs and Centaurs were originally similar but evolutionary processes have altered them differently, hence their color diversity. Most research has focused on the latter hypothesis, offering little improvement to our understanding of the color distributions. Luu \& Jewitt (1996) proposed that the competition between a reddening effect of the irradiation of surface ices (Thompson et al. 1987) and a bluing effect due to the collisionally induced resurfacing of fresh non-irradiated ices might generate the observed surface colors. The same authors, however, rejected this model as being the primary cause of the color diversity, owing to the lack of predicted rotational color variations (Jewitt \& Luu 2001). On the basis of the same processes, Gil-Hutton (2002) proposed a 
more complex treatment of the irradiation process, by assuming an intricate structure of differently irradiated subsurface layers. However, the collisional resurfacing effects became very hard to model, thus making it very hard to provide testable predictions. Later, Thébault \& Doressoundiram (2003) showed that the collisional energies involved in different parts of the Kuiper belt did not corroborate the possible link between surface colors and non-disruptive collisions.

Delsanti et al. (2004) refined the first of the aforementioned models by considering the effects of possible cometary activity triggered by collisions, and a size/gravity-dependent resurfacing. Cometary activity can modify the surface properties through the creation of a neutral-color dust mantle. Jewitt (2002) suggested that this process could explain why no JFCs are found with the ultra-red surfaces seen in about half of the Centaurs. It has also been proposed that the sublimation loss of surface ice from a mixture with red materials may be sufficient to make the red material undetectable at visible wavelengths (Grundy 2009). These might explain the Centaur color bimodality, as long as all were red when migrating inwards from the Kuiper belt. Although promising, these models did not provide an explanation of the color bimodality of Centaurs, as they fail to reproduce the bluest colors observed and their frequency.

\section{Motivation for this work}

We find it puzzling that the objects with both perihelia and semimajor axes between Jupiter and Neptune's orbits, the Centaurs - by definition-, display a different color distribution from physically and chemically similar objects with semi-major axes slightly beyond Neptune's orbit, as in the case of scattered disk objects (SDOs), for instance, or any other KBOs. There is no evident physical consideration that would explain an apparently sudden "transition" in surface color behavior (from bimodal to unimodal) precisely at Neptune's orbital semi-major axis $a_{\mathrm{N}}=$ 30.07 AU. This difference between Centaurs and KBOs is particularly puzzling because there is neither a sharp dynamical separation between them (the definition is somewhat arbitrary), nor a clearly identified family of KBOs that could be their origin. Although SDOs are frequently considered as the main source of Centaurs, we note that Neptune Trojans, Plutinos, and classical KBOs have also been found to be viable contributors (Horner \& Lykawka 2010; Yu \& Tremaine 1999; Volk \& Malhotra 2008, respectivelly). Furthermore, Centaurs possess short dynamical lifetimes of $\sim 5 \times 10^{5}-3 \times 10^{7} \mathrm{yr}$ before being injected as JFCs or ejected again into the outer solar system (Horner et al. 2004). If some surface evolution mechanism, dependent on heliocentric distance, is responsible for the bimodal behavior of Centaurs, it must be acting extremely rapidly such that no intermediate colors are ever seen among them. Apart from surface color bimodality, the most distinctive characteristic of Centaurs compared to "other" KBOs is their small size. Known KBOs are mostly larger than Centaurs, simply because they are more distant and thus smaller objects are harder to detect.

In this work, we address the issue of the color distributions of Centaurs and KBOs. We present new data on seven intrinsically faint (thus small) KBOs and three Centaurs, combined with a new compilation of 253 published $B-R$ colors, and available $m_{R}(1,1, \alpha)$ magnitudes, or $H_{R}(\alpha)$, i.e. absolute magnitudes that have not been corrected for phase effects, and some identified spectral features. We study this large sample of colors (including objects from all dynamical families) versus absolute magnitude as a proxy for size, with the implicit assumption that surface colors are independent of dynamical classification. We present
Table 1. Filters specifications.

\begin{tabular}{cccccc}
\hline \hline & \multicolumn{2}{c}{$8.2 \mathrm{~m}$ Subaru } & & \multicolumn{2}{c}{ UH 2.2 m } \\
\cline { 2 - 3 } \cline { 5 - 6 } Filter & \multicolumn{2}{c}{ Wavelength $(\AA)$} & & \multicolumn{2}{c}{ Wavelength $(\AA)$} \\
\hline & Center & Width & & Center & Width \\
$B$ & 4400 & 1080 & & 4480 & 1077 \\
$R$ & 6600 & 1170 & & 6460 & 1245 \\
\hline
\end{tabular}

the most relevant results, namely those found in $B-R$ vs. $H_{R}(\alpha)$ space.

\section{Observations and data reduction}

Observations of $7 \mathrm{KBOs}$ and 1 Centaur were taken at the 8.2 m Subaru telescope, on 2008-07-02, using 0.'206/pix FOCAS camera in imaging mode with $2 \times 2$ binning $(2$ CCDs of $2048 \times 4096$ pixels, Kashikawa et al. 2002). Weather was clear with seeing $\sim 0.7^{\prime \prime}$. We used the University of Hawaii UH 2.2 m telescope, to observe 2 Centaurs on 2008-09-29, with the 0 .'22/pixel Tektronix $2048 \times 2048$ pixels CCD camera. Weather was clear with seeing $\sim 0.9^{\prime \prime}$. Both telescopes are on Mauna Kea, Hawaii, USA. Images from both instruments were processed using IRAF's CCDRED package following the standard techniques of median bias subtraction and median flat-fielding normalization.

Standard calibration was made observing Landolt standard stars (Landolt 1992) at different airmasses for each filter, obtaining the corresponding zeropoints, solving by non-linear leastsquare fits the transformation equations, directly in order of $R$ and $(B-R)$, using IRAF's PHOTCAL package. The characteristics of the filters used on each telescope were essentially equivalent (Table 1). Subaru's data was calibrated using the Landolt standard stars 107-612, PG1047+003B, 110-230, Mark A2, and 113-337, which were observed repeatedly at different airmasses. The data acquired at the UH2.2 m were calibrated, analogously, using the stars 92-410, 92-412, 94-401, 94-394, PG2213-006A, and PG2213-006B. These stars have high photometric accuracy and colors close to those of the Sun. We used the typical extinction values for Mauna Kea of $k_{B}=0.19$ and $k_{R}=0.09$ (Krisciunas et al. 1987, and CFHT Info Bulletin \#19). All fits had residuals rms $<0.02$, which were added quadratically to the photometric error in each measurement. Targets were observed twice in $B$ band and twice in $R$ band, to avoid objects trailing in one long exposure. Each of the two $B$ or $R$ exposures were coadded centered on the object, and also co-added centered on the background stars. The former were used to measure the object, the latter to compute the growth-curve correction. The time and airmass of observation were computed for the center of the total exposure time. We applied growth-curve correction techniques to measure the target's magnitudes using IRAF's MKAPFILE task (for details, see Peixinho et al. 2004). A description of the observation and results are shown in Table 2.

\section{Compilation of data}

We compiled the visible colors of 290 objects (KBOs, Centaurs, and Neptune Trojans) for which the absolute magnitude in either $R$ or $V$ band was accessible (e.g. with individual magnitudes and observing date available), and surface spectra information for 48 objects, as published in the literature to date (Feb. 2012). We computed the absolute magnitude $H_{R}(\alpha) \equiv m_{R}(1,1, \alpha)=$ $R-5 \log (r \cdot \Delta)$, where $R$ is the $R$-band magnitude, and $r$ and $\Delta$ 
N. Peixinho et al.: The bimodal colors of Centaurs and small Kuiper belt objects

Table 2. Observational circumstances and photometric results of this work's data.

\begin{tabular}{|c|c|c|c|c|c|c|c|c|c|c|}
\hline \multicolumn{2}{|c|}{$\overline{\text { Object }}$} & Dyn. class* ${ }^{*}$ & Telescope & UT date & $r[\mathrm{AU}]$ & 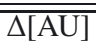 & $\overline{\alpha \alpha\left[^{\circ}\right]}$ & $R$ & $\overline{B-R}$ & $H_{R}(\alpha)$ \\
\hline (130391) & $2000 \mathrm{JG}_{81}$ & $2: 1$ & Subaru & 20080702UT07:24:58 & 34.073 & 34.817 & 1.2 & $23.12 \pm 0.03$ & $1.42 \pm 0.06$ & $7.75 \pm 0.06$ \\
\hline (136120) & $2003 \mathrm{LG}_{7}$ & $3: 1$ & Subaru & 20080702UT09:42:53 & 32.815 & 33.659 & 1.0 & $23.54 \pm 0.05$ & $1.27 \pm 0.09$ & $8.32 \pm 0.05$ \\
\hline 560) & $2003 \mathrm{QZ}_{91}$ & SDO & Subaru & 20080702UT13:08:33 & 25.849 & 26.509 & 1.7 & $22.48 \pm 0.03$ & $1.30 \pm 0.05$ & $8.30 \pm 0.03$ \\
\hline & $2006 \mathrm{RJ}_{103}$ & Nep. Trojan & Subaru & 20080702UT14:07:50 & 30.760 & 30.534 & 1.9 & $22.27 \pm 0.02$ & $1.90 \pm 0.04$ & $7.40 \pm 0.02$ \\
\hline & $2006 \mathrm{SQ}_{372}$ & SDO & Subaru & 20080702UT11:45:34 & 23.650 & 24.287 & 1.9 & $21.55 \pm 0.02$ & $1.78 \pm 0.03$ & $7.71 \pm 0.05$ \\
\hline & $2007 \mathrm{JK}_{43}$ & SDO & Subaru & 20080702UT08:08:13 & 23.113 & 23.766 & 1.9 & $20.73 \pm 0.02$ & $1.40 \pm 0.03$ & 0.02 \\
\hline & $2007 \mathrm{NC}_{7}$ & SDO & Subaru & 20080702UT11:30:49 & 20.090 & 20.916 & 1.7 & $21.19 \pm 0.02$ & $1.28 \pm 0.03$ & $8.07 \pm 0.02$ \\
\hline (281371) & $2008 \mathrm{FC}_{76}$ & Cent & Subaru & 20080702UT11:13:05 & 11.119 & 11.793 & 3.8 & $19.79 \pm 0.02$ & $1.76 \pm 0.02$ & $9.18 \pm 0.04$ \\
\hline & $2007 \mathrm{RH}_{283}$ & Cent & $\mathrm{UH} 2.2 \mathrm{~m}$ & 20080929UT12:43:47 & 17.081 & 17.956 & 1.6 & $20.85 \pm 0.03$ & $1.20 \pm 0.05$ & \\
\hline & $2007 \mathrm{RH}_{283}$ & Cent & $\mathrm{UH} 2.2 \mathrm{~m}$ & 20080929UT12:57:51 & 17.081 & 17.956 & 1.6 & $20.90 \pm 0.03$ & $=0.06$ & \\
\hline & $\begin{array}{l}\text { mean... } \\
2007 \mathrm{UM}_{126}\end{array}$ & Cent & $\mathrm{UH} 2.2 \mathrm{~m}$ & 2008 & 10.191 & 11.177 & 0.9 & $20.43 \pm 0.03$ & $\begin{array}{l}1.24 \pm 0.07 \\
1.21 \pm 0.05\end{array}$ & \\
\hline & $2007 \mathrm{UM}_{126}$ & Cent & $\mathrm{UH} 2.2 \mathrm{~m}$ & 20080929UT09:06:41 & 10.191 & 11.177 & 0.9 & $20.53 \pm 0.03$ & $0.92 \pm 0.04$ & \\
\hline & $2007 \mathrm{UM}_{126}$ & Cent & $\mathrm{UH} 2.2 \mathrm{~m}$ & 20080929UT09:16:17 & 10.191 & 11.177 & 0.9 & $20.38 \pm 0.02$ & $\begin{array}{l}1.12 \pm 0.04 \\
1.08+0.10\end{array}$ & 10. \\
\hline
\end{tabular}

Notes. ${ }^{(*)}$ Dynamical classes are: Centaur, scattered disk object (SDO), Neptune Trojan (object located in 1:1 mean motion resonance with Neptune), 2:1, and 3:1 (objects located in 2:1 or 3:1 mean motion resonance with Neptune, respectively). For details of our classification, see Appendix A.

are the helio- and geocentric distances in AU, respectively. In this compilation, 253 objects have $B-R$ colors available, which are the focus of this paper (see Table 3), and 48 also have spectral information. The description of the compilation method is presented in Appendix A. Sun-Object-Earth phase angles $\alpha$ are, typically, smaller than $1.5^{\circ}$ for KBOs and less than $4^{\circ}$ for Centaurs. Measurements of magnitude dependences on the phase angle for these objects, i.e. phase coefficients $\beta\left[\mathrm{mag} /{ }^{\circ}\right]$, are scarce but, so far, do not show any evidence of extreme variability, showing instead an average value of $\beta=0.11 \pm 0.05$ (Belskaya et al. 2008). Looking at the linear approximation $H_{R}\left(\alpha=0^{\circ}\right) \approx H_{R}(\alpha)-\alpha \beta$, we can see that by not correcting the absolute magnitude for phase effects we slightly overestimate it. We deal with this issue in Sect. 5.

Recent works have shown that there is no strong correlation between object diameter $D$ and geometric albedo $p_{V}$, nor between geometric albedo $p_{V}$ and absolute magnitude $H_{R}$ (Stansberry et al. 2008; Santos-Sanz et al. 2012; Vilenius et al. 2012; Mommert et al. 2012). However, on the basis of the 74 diameter and albedo measurements of Centaurs and KBOs made using Herschel and/or Spitzer telescopes, published in the aforementioned works, we verified that $H_{R}$ and $D$ correlate very strongly with a Spearman-rank correlation of $\rho=-0.92_{-0.02}^{+0.03}$, with a significance level $S L \ll 0.01 \%$ (error bars computed using bootstraps, for details see Doressoundiram et al. 2007). Consequently, absolute magnitude is a very good proxy of size.

\section{An $\mathcal{N}$-shaped doubly bimodal structure}

In Fig. 1, we plot the $R$-band absolute magnitude $H_{R}(\alpha)$ (proxy of an object's size) against $B-R$ color for all $(n=253)$ objects in our database. The cloud of points forms a recognizable $\mathcal{N}$ shape with an apparent double bimodal structure in color. The smaller objects (upper part of the plot) show a bimodal $B-R$ distribution. Although apparently dominated by Centaurs, this bimodal distribution also includes KBOs of similar $H_{R}(\alpha)$, which suggests that the bimodal structure in $B-R$ color is a property of the smaller objects in general, regardless of their dynamical family. This bimodality appears to disappear for objects with $H_{R}(\alpha) \lesssim 7$, where the $B-R$ color distribution seems unimodal. Most interestingly, we note that towards the larger objects (lower part of the plot) the colors appear to follow another bimodal distribution, with

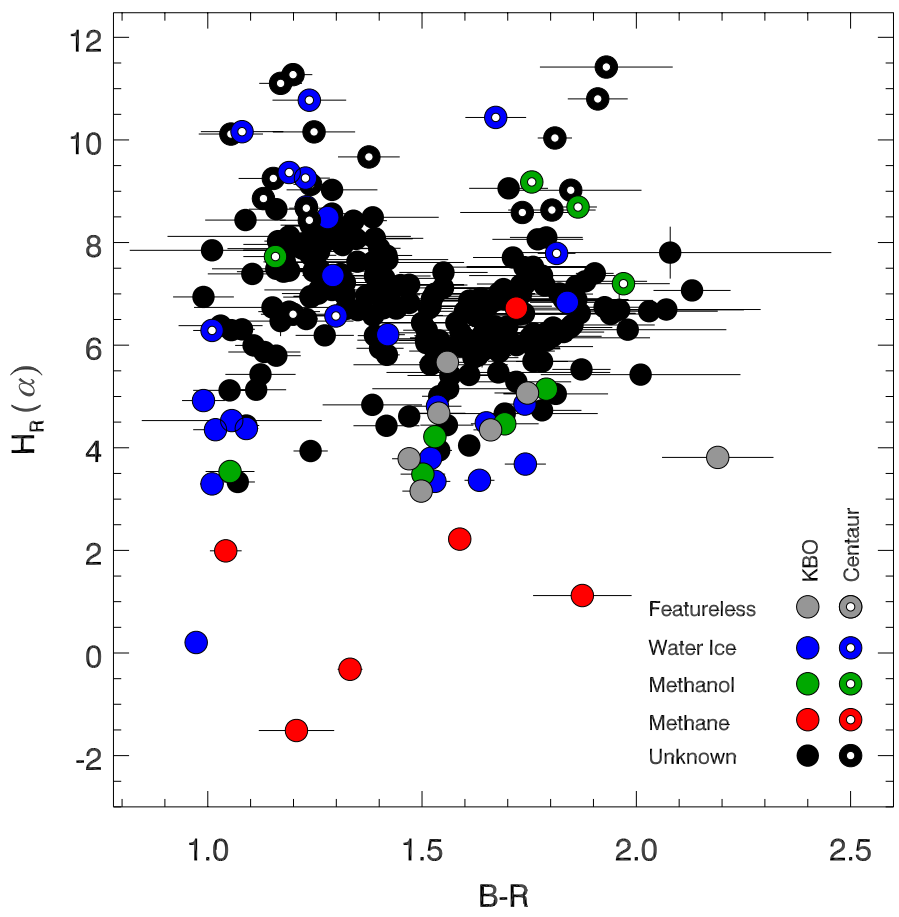

Fig. 1. $B-R$ vs. $H_{R}(\alpha)$ plot of all 253 objects. KBOs are represented by solid circles and Centaurs by white dotted solid circles. Objects with $H_{R}(\alpha) \geqslant 6.8$ separate into two color groups with a "gap" centered at $B-R \sim 1.60$. Objects with $H_{R}(\alpha) \leqslant 5.0$ also show statistical evidence of a separation into two-color groups but with a "gap" centered at $B-$ $R \sim 1.25$. Objects spectra with known features of water ice, methane, methanol, and featureless spectra, are coded using colors as described in the legend. There is no obvious/clear connection between $B-R$ colors and the presence of spectral features.

the gap between the two groups shifted towards the blue with respect to the "small" object bimodality. This new "large" object bimodality is explicitly reported for the first time.

When performing hypothesis testing, one should adopt a critical value of significance $\alpha$. The value $\alpha$ is the maximum probability (risk) we are willing to take in rejecting the null hypothesis $H_{0}$ (i.e. to claim no evidence of bimodality) when it 
is actually true (i.e. data is truly bimodal/multimodal) which is also called type I error probability. This value is often a source of debate, as are the theories of hypothesis testing themselves (e.g. Lehmann 1993). The decision relies mostly upon whether the effects of a right or wrong decision are of either practical importance or consequence. The paradigm is that by diminishing the probability of wrongly rejecting a null hypothesis (e.g. deciding that bimodality is found when bimodality is not present in the parent population), we increase the probability of wrongly accepting the null hypothesis (i.e. deciding on unimodality when bimodality is in fact present), also called type II error probability, or risk factor $\beta$. Some authors and/or research fields consider that there is only sufficient evidence against $H_{0}$ when the achieved significance level is $S L<0.3 \%$, i.e. using $\alpha=0.3 \%$ (the $3 \sigma$ Gaussian probability), whereas others require even $\alpha=0.0003 \%(6 \sigma)$. This might be a criterion for rejecting $H_{0}$ but is not a very useful "rating" of the evidence against $H_{0}$, which is what we are implicitly doing. We rate the evidence against $H_{0}$ following a common procedure in statistics: we assume that $S L<5 \%$ implies that there is reasonably strong evidence against $H_{0}, S L<2.5 \%$ that there is strong evidence against $H_{0}$, and $S L<1 \%$ that there is very strong evidence against $H_{0}$ (e.g. Efron \& Tibshirani 1993). We also add the common procedure in physics that $S L<0.3 \%$ represents clear evidence against $H_{0}$. Furthermore, for better readability, we employ throughout this work the term "evidence for bimodality" instead of the statistically correct term "evidence against unimodality".

Using the R software's (version 2.14.1; R Development Core Team 2011) Dip Test package (Hartigan 1985; Hartigan \& Hartigan 1985; Maechler 2011), we test the null hypothesis $H_{0}$ that "the sample is consistent with an unimodal parent distribution" over all objects in the $B-R$ vs. $H_{R}(\alpha)$ space, against the alternative hypothesis $H_{1}$ that "the sample is inconsistent with an unimodal parent distribution" (hence it is either bimodal or multimodal). The full sample, in spite of the apparent two spikes, shows no strong evidence against color unimodality neither with $(n=253, S L=17 \%)$ nor without $(n=224$, $S L=41 \%$ ) Centaurs (see Fig. 2a). The Centaur population $(n=29)$ shows strong evidence against unimodality at $1.6 \%$. Removing the 3 brightest Centaurs (with $H_{R}(\alpha) \gtrsim 6.6$ ) improves this significance to $0.3 \%$. To refine the analysis and test different ranges in $H_{R}(\alpha)$, we applied the Dip Test to sub-samples using a running cutoff in $H_{R}(\alpha)$ that was shifted by 0.1 mag between consecutive tests.

Bimodal distribution of "small" objects: we performed iterative Dip Tests with a $H_{R \text { : cut }}$ starting at the maximum $H_{R}(\alpha)$ value, and decreasing in steps of $0.1 \mathrm{mag}$; in each iteration, we applied the test to those objects above the cutoff line (i.e. with $\left.H_{R}(\alpha) \geqslant H_{R \text { : cut }}\right)$. We stopped shifting $H_{R \text { : cut when we detected }}$ the maximum of evidence against unimodality (i.e. a minimum of significance level), henceforth accepted the alternate hypothesis that "the distribution is bimodal/multimodal") Evidence of bimodality at significance levels better than $5 \%$ start to be seen for objects with $H_{R}(\alpha) \geqslant 7.1$. This evidence peaks at a significance of $0.1 \%$ for the 124 faint objects with $H_{R}(\alpha) \geqslant 6.8$.

We propose that the visible surface color distribution of (non-active) icy bodies of the outer solar system depends only on object size, and is independent of their dynamical classification. No mechanism has yet been found to explain the color bimodality only for Centaurs. However, since this mechanism might exist, even if it has not yet been found, we re-analyze the sample removing the Centaurs. Naturally, the sampling of the smaller

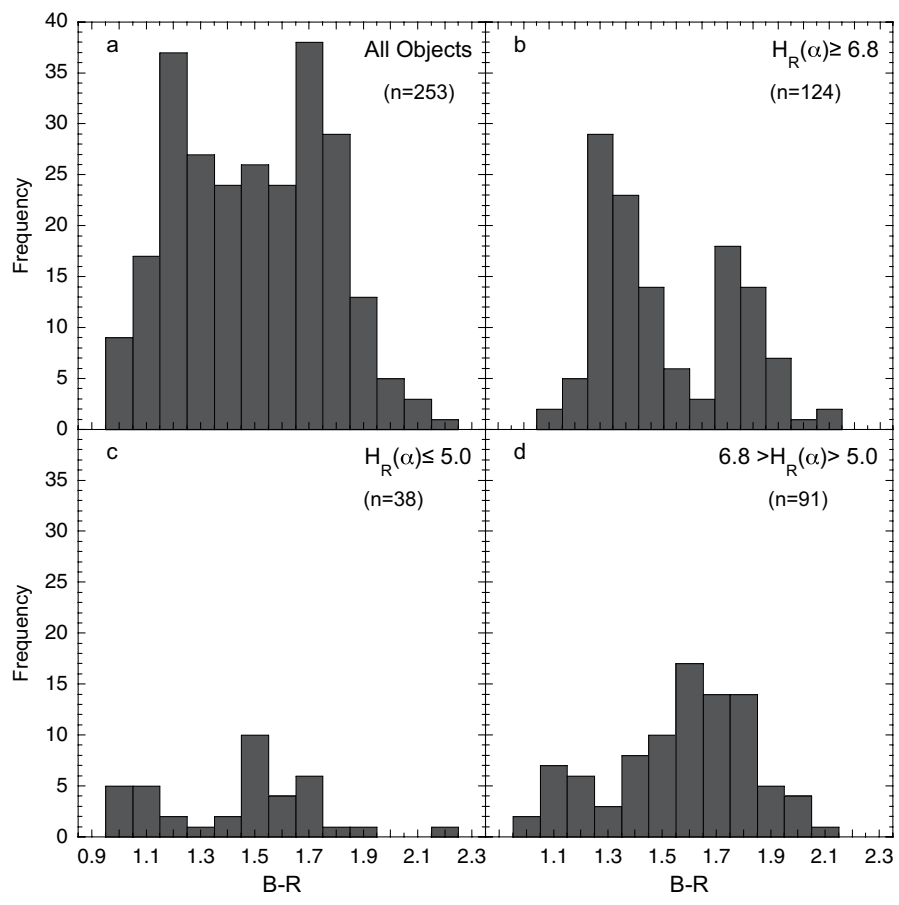

Fig. 2. Histograms of $B-R$ colors from selected $H_{R}(\alpha)$ ranges: a) all the 253 objects. Taken globally do not exhibit statistical evidence for bimodality, which was known to exist among Centaurs. b) The 124 "small" objects, with $H_{R}(\alpha) \geqslant 6.8$. Evidence for bimodal behavior is clear and still present when removing Centaurs. c) The 38 "large" objects, with $H_{R}(\alpha) \leqslant 5.0$. A bimodal behavior is shown but it loses the statistical significance without the "Haumea collisional family" objects. d) The 91 "intermediate" size objects, $6.8>H_{R}(\alpha)>5.0$. Regardless of the apparent small gap at $B-R \sim 1.3$ there is no statistical evidence for two separate groups.

objects diminishes considerably, hence reducing the statistical significance against the null hypothesis (i.e. increases the probability of observing two groups on a purely random distribution of colors). Nonetheless, the 98 remaining objects with $H_{R}(\alpha) \geqslant 6.8$ show evidence of bimodality at a significance level of $3.5 \%$, reaching a significance minimum of $1.8 \%$ for the 165 objects with $H_{R}(\alpha) \geqslant 5.8$. In both cases, the "gap" is centered around $B-R \sim 1.60$ (see Figs. 1 and $2 \mathrm{~b}$ ).

Bimodal distribution of "large" objects: we test the brightest part of the sample using a cutoff limit starting at the minimum $H_{R}(\alpha)$ value; we consider objects below the cutoff (i.e. brighter than $\left.H_{R \text { : cut }}\right)$ and shift it up in steps of $0.1 \mathrm{mag}$. We find very strong evidence against unimodality for objects with $H_{R}(\alpha) \lesssim$ $5.0(S L=0.9 \%)$. Data still shows reasonably strong evidence against unimodality for objects up to $H_{R}(\alpha) \lesssim 5.6$. The "gap" is located at $B-R \sim 1.25$. There are no Centaurs in this brightness range. Explicitly, evidence of "large" object bimodality has not been previously reported (see Figs. 1 and 2c). Removing from the sample the 7 objects belonging to the "Haumea collisional family" (Brown et al. 2007b; Snodgrass et al. 2010), which are all clustered on the lower left "leg" of the $\mathcal{N}$ shape, erases the statistical evidence against the null hypothesis, even if still suggestive to the eye. Therefore, with the present data sample, the "evidence of bimodality" among bright KBOs cannot be stated to be independent of the peculiar properties of the Haumea collisional family. 
The "intermediate" size continuum: the 91 objects with $6.8>$ $H_{R}(\alpha)>5.0$, which include 3 Centaurs, do not show evidence against a unimodal behavior ( $S L=98.0 \%$ ), even if a small gap seems suggestive to the eye (see Figs. 1 and 2d). However, statistically, their inclusion in the fainter group does not decrease the significance below the "strong evidence against unimodality", i.e. $S L=2.5 \%$ (see Figs. 1 and 2d). On the other hand, if added to the "large" objects the statistical evidence for the bimodality of "large" objects does not hold.

To check for the effects of not correcting $H_{R}(\alpha)$ for phaseangle effects we performed Monte Carlo simulations. First, we computed all the possible $\alpha$ values and their probability distribution for an "average" Centaur with a semi-major axis $a=15$ AU. The maximum $\alpha$ is $3.8^{\circ}$ and the median value is $3.2^{\circ}$. Analogously, we did the same for a KBO with $a=40 \mathrm{AU}$. The maximum $\alpha$ is $1.4^{\circ}$ and the median value is $1.2^{\circ}$. Therefore, on average, our absolute magnitudes might be overestimated by $\Delta H_{R} \approx 0.35$ for Centaurs and by $\Delta H_{R} \approx 0.13$ for KBOs. Simulating 1000 "phase-corrected" $H_{R}$ data-samples, we found that following the probability distribution of the corresponding $\alpha$ angles did not alter any of the results obtained using simply $H_{R}(\alpha)$.

\section{Interpretation}

Our analysis shows that the $B-R$ colors of Centaurs and KBOs when plotted as a function of $H_{R}(\alpha)$ display an $\mathcal{N}$-shaped, double, bimodal behavior. The color distribution seems to depend on object size (intrinsic brightness) instead of dynamical family. Using the brightness-size-albedo relation $D_{\mathrm{km}}=$ $2 \sqrt{2.24 \times 10^{16} \times 10^{0.4\left(H_{R \odot}-H_{R}\right) / p_{R}}}$, with solar $H_{R \odot}=-27.10$, the main issue is to choose a canonical geometric albedo value $p_{R}$. Recent works (Stansberry et al. 2008; Santos-Sanz et al. 2012; Vilenius et al. 2012; Mommert et al. 2012) measured a wide range of albedo values, for each dynamical family, in some cases far from the 0.04 value previously assumed based on comet studies. As we needed only a rough estimate of the size ranges, we selected the average value of $p_{R}=0.09$. Using this parameter, objects with diameters $165 \lesssim D_{\mathrm{km}} \lesssim 380$ follow a rather continuous range of $B-R$ colors.

Visible and near-infrared (NIR) spectroscopy for about 75 bright objects (Barucci et al. 2011, for a review) also indicates that the surface compositions of KBOs and Centaurs is very diverse. The largest objects are coated in methane ice, while intermediate-size objects display water-ice features, sometimes with traces of other volatiles. Small KBOs generally have featureless spectra. The presence of volatiles on the surface of an object may be related to its ability to retain them, i.e. to its size and temperature (Schaller \& Brown 2007). It should also depend on the subsequent irradiation history (Brown et al. 2011). However, no correlation can be made to date between visible colors and NIR spectral properties. For example, two objects of comparable size, Quaoar and Orcus, both exhibit water icedominated surfaces but have, respectively, very red and neutral visible colors (Delsanti et al. 2010).

Objects smaller than $\sim 100-150 \mathrm{~km}$, including most of the known Centaurs, are believed to be fragments from the collision of larger objects (Pan \& Sari 2005). Predicting the properties of these fragments is a complex task, but the field shows promising advances (for a review, see Leinhardt et al. 2008). An immediate hypothesis is that red and neutral objects are the only possible outcomes of a disruptive collision. Thermal evolution modeling suggests that KBOs, especially large ones, should have a layered structure, including some liquid water leading to a complete differentiation of the object (Merk \& Prialnik 2006; Guilbert-Lepoutre et al. 2011). A catastrophic collision could result in the formation of fragments with very different properties, depending on whether they come from the core of the parent body, or its mantle, or some subsurface layers. However, our current knowledge of KBO internal properties and evolution is still incipient to support or discard such hypothesis. In addition, it is hard to understand why objects with $B-R \sim 1.6$ (in the gap of the small object's bimodal distribution) should not exist. Maybe their relative number is so small compared to the neutral and red groups that it is extremely difficult to observe them, leading to other puzzling questions. Research on these aspects should be encouraged. In particular, the detection and measurement of many more small objects - KBOs and Centaurs - could help us to further constrain their color distribution and other properties. The objects in the "intermediate" $H_{R}(\alpha)$ range $\left(6.8>H_{R}(\alpha)>5.0\right)$ seem unimodally distributed in $B-R$ color; they might represent a transition phase between the two bimodal distributions. These medium-sized objects are probably too large to be remnants from disruptive collisions, and too small to have recently undergone cryovolcanic activity (their properties may not even have differentiated). They might, actually, represent the only group where the outcomes of the combined effects of different birthplaces, space weathering, and thermal processing can be studied or analyzed.

The evidence of a bimodal distribution among the largest objects is also puzzling. These have been supposedly the most well-studied objects, yet the evidence for a bimodal distribution of their surface colors has never been reported. Nonetheless, removing the 7 Haumea collisional family objects from our sample we no longer find evidence against an unimodal distribution, even if it is apparent to the eye. This issue should be further analyzed in great detail when a larger sample is available.

In this work, we confirm that there is no noticeable link between the surface composition of an object and its visible colors. Objects hosting water ice are evenly distributed both among large and small objects, and among red and blue ones. When it comes to volatiles such as methane $\left(\mathrm{CH}_{4}\right)$ or methanol $\left(\mathrm{CH}_{3} \mathrm{OH}\right)$, we have found that they are also distributed among all groups, although they might be more difficult to detect for small/fainter objects. We nonetheless find a cluster of featureless objects among the red group of large objects: these might represent the most irradiated/oldest surfaces in the overall population. Therefore, it seems that a simple explanation such as the model of atmospheric escape proposed by Schaller \& Brown (2007) might not be sufficient to explain the colors and compositions of KBOs. The reason why they evolved into two different color groups can be very complex, and should involve different thermal, collisional, irradiation histories, in addition to possibly different birthplaces.

\section{Summary}

We have analyzed the $B-R$ color distribution as a function of $H_{R}(\alpha)$ magnitude for 253 Centaurs and KBOs, including 10 new measurements, and with the information on their NIR spectral features. Using the known diameters, $D$, and albedos, $p_{V}$, of 74 of these objects we verify that $H_{R}$ and $D$ correlate very strongly $\left(\rho=-0.92_{-0.02}^{+0.03}, S L \ll 0.01 \%\right)$, validating $H_{R}$ as a good proxy of size. Furthermore, through simulations, we show that 
not correcting $H_{R}(\alpha)$ to $H_{R}\left(\alpha=0^{\circ}\right)$ does not change any of the global results. Our analysis shows that:

1. The $B-R$ vs. $H_{R}(\alpha)$ color distribution is $\mathcal{N}$-shaped, indicating that $B-R$ colors are probably dominated by a size effect independent from dynamical classification.

2. Small objects, including both KBOs and Centaurs, display a bimodal structure of $B-R$ colors at a $0.1 \%$ significance level (i.e. objects with $H_{R}(\alpha) \geqslant 6.8$, or $D_{\mathrm{km}} \lesssim 165$, assuming that $\left.p_{R}=0.09\right)$ with the "gap" centered at $B-R \sim 1.60$. Removing Centaurs from the sample greatly reduces the sampling of small objects, reducing also the significance of the result to $3.8 \%$.

3. Large objects also appear to have a bimodal color distribution, with a minimum significance of $0.9 \%$, for $H_{R}(\alpha) \lesssim$ $5.0\left(D_{\mathrm{km}} \gtrsim 380\right.$, assuming that $\left.p_{R}=0.09\right)$, and a color "gap" centered at $B-R \sim 1.25$. Reasonable evidence of this bimodality starts when considering only objects with $H_{R}(\alpha) \lesssim 5.6\left(D_{\mathrm{km}} \gtrsim 290\right)$, dropping below the critical $5 \%$ when reaching $H_{R}(\alpha) \lesssim 4.4\left(D_{\mathrm{km}} \gtrsim 500\right)$. However, this behavior seems dominated by the presence of 7 Haumea collisional family objects, which "cluster" at the lower left edge of the $\mathcal{N}$-shape. Once removed, there is no statistical evidence against compatibility with a random unimodal distribution for the larger KBOs.

4. Intermediate-size objects do not show incompatibility with a continuum of $B-R$ colors (i.e. $6.8>H_{R}(\alpha)>5.0$, or $165 \lesssim D_{\mathrm{km}} \lesssim 380$, assuming $p_{R}=0.09$ ). These objects seem too large to be the remnants of disruptive collisions and too small to display cryovolcanic activity. They might be the best targets for study of the combined effects of different birthplaces, different space weathering, and different thermal processing. Further studies are encouraged.

5. Inspecting the NIR spectral properties against $B-R$ colors shows that there is no obvious link between the colors and the chemical compositions of the objects' surfaces.

Acknowledgements. The authors thank Rachel Stevenson, Megan Bagley, and Takashi Hatori for assisting with the observations at Subaru telescope. N.P. was partially supported by NASA's Origins grant to D. Jewitt, by the European Social Fund, by the Portuguese Foundation for Science and Technology (FCT, ref.: BPD/18729/2004), and by the transnational cooperation agreement FCT-Portugal/CNRS-France (ref.: 441.00). A.G.L. was supported by a NASA Herschel grant to D. Jewitt. P.L. is grateful for financial support from a Michael West Fellowship and from the Royal Society in the form of a Newton Fellowship.

\section{References}

Barkume, K. M., Brown, M. E., \& Schaller, E. L. 2008, AJ, 135, 55

Barucci, M. A., Doressoundiram, A., Tholen, D., Fulchignoni, M., \& Lazzarin, M. 1999, Icarus, 142, 476

Barucci, M. A., Romon, J., Doressoundiram, A., \& Tholen, D. J. 2000, AJ, 120, 496

Barucci, M. A., Morea Dalle Ore, C., Alvarez-Candal, A., et al. 2010, AJ, 140, 2095

Barucci, M., Alvarez-Candal, A., Merlin, F., et al. 2011, Icarus, 214, 297

Belskaya, I. N., Levasseur-Regourd, A.-C., Shkuratov, Y. G., \& Muinonen,

K. 2008, Surface Properties of Kuiper Belt Objects and Centaurs from

Photometry and Polarimetry, eds. M. A. Barucci, H. Boehnhardt, D. P.

Cruikshank, A. Morbidelli, \& R. Dotson, 115

Boehnhardt, H., Tozzi, G. P., Birkle, K., et al. 2001, A\&A, 378, 653

Boehnhardt, H., Delsanti, A., Barucci, A., et al. 2002, A\&A, 395, 297

Brown, R. H., Cruikshank, D. P., \& Pendleton, Y. 1999, ApJ, 519, L101

Brown, M. E., Barkume, K. M., Blake, G. A., et al. 2007a, AJ, 133, 284

Brown, M. E., Barkume, K. M., Ragozzine, D., \& Schaller, E. L. 2007b, Nature, 446, 294

Brown, M. E., Schaller, E. L., \& Fraser, W. C. 2011, ApJ, 739, L60
Cruikshank, D. P., Roush, T. L., Bartholomew, M. J., et al. 1998, Icarus, 135, 389

Delsanti, A. C., Boehnhardt, H., Barrera, L., et al. 2001, A\&A, 380, 347

Delsanti, A., Hainaut, O., Jourdeuil, E., et al. 2004, A\&A, 417, 1145

Delsanti, A., Merlin, F., Guilbert-Lepoutre, A., et al. 2010, A\&A, 520, A40

DeMeo, F. E., Barucci, M. A., Merlin, F., et al. 2010, A\&A, 521, A35

Doressoundiram, A., Barucci, M. A., Romon, J., \& Veillet, C. 2001, Icarus, 154, 277

Doressoundiram, A., Peixinho, N., de Bergh, C., et al. 2002, AJ, 124, 2279

Doressoundiram, A., Barucci, M. A., Tozzi, G. P., et al. 2005a, Planet. Space Sci., 53,1501

Doressoundiram, A., Peixinho, N., Doucet, C., et al. 2005b, Icarus, 174, 90

Doressoundiram, A., Peixinho, N., Moullet, A., et al. 2007, AJ, 134, 2186

Dotto, E., Barucci, M. A., Boehnhardt, H., et al. 2003, Icarus, 162, 408

Efron, B., \& Tibshirani, R. J. 1993, An Introduction to the Bootstrap (Chapman \& Hall/CRC)

Fernandez, J. A. 1980, MNRAS, 192, 481

Ferrin, I., Rabinowitz, D., Schaefer, B., et al. 2001, ApJ, 548, L243

Fornasier, S., Doressoundiram, A., Tozzi, G. P., et al. 2004, A\&A, 421, 353

Gil-Hutton, R. 2002, Planet. Space Sci., 50, 57

Gladman, B., Marsden, B. G., \& Vanlaerhoven, C. 2008, Nomenclature in the Outer Solar System, eds. M. A. Barucci, H. Boehnhardt, D. P. Cruikshank, A. Morbidelli, \& R. Dotson, 43

Green, S. F., McBride, N., O'Ceallaigh, D. P., et al. 1997, MNRAS, 290, 186

Grundy, W. M. 2009, Icarus, 199, 560

Grundy, W. M., Buie, M. W., \& Spencer, J. R. 2005, AJ, 130, 1299

Guilbert, A., Alvarez-Candal, A., Merlin, F., et al. 2009a, Icarus, 201, 272

Guilbert, A., Barucci, M. A., Brunetto, R., et al. 2009b, A\&A, 501, 777

Guilbert-Lepoutre, A., Lasue, J., Federico, C., et al. 2011, A\&A, 529, A71

Gulbis, A. A. S., Elliot, J. L., \& Kane, J. F. 2006, Icarus, 183, 168

Hainaut, O. R., \& Delsanti, A. C. 2002, A\&A, 389, 641

Hainaut, O. R., Delahodde, C. E., Boehnhardt, H., et al. 2000, A\&A, 356, 1076

Hartigan, P. M. 1985, Appl. Stat., 34, 320

Hartigan, J. A., \& Hartigan, P. M. 1985, Ann. Stat., 13, 70

Horner, J., \& Lykawka, P. S. 2010, MNRAS, 402, 13

Horner, J., Evans, N. W., \& Bailey, M. E. 2004, MNRAS, 354, 798

Jewitt, D. C. 2002, AJ, 123, 1039

Jewitt, D., \& Luu, J. 1993, Nature, 362, 730

Jewitt, D., \& Luu, J. 1998, AJ, 115, 1667

Jewitt, D. C., \& Luu, J. X. 2001, AJ, 122, 2099

Kashikawa, N., Aoki, K., Asai, R., et al. 2002, PASJ, 54, 819

Kern, S. D., McCarthy, D. W., Buie, M. W., et al. 2000, ApJ, 542, L155

Kowal, C. T., Liller, W., \& Chaisson, L. J. 1977, IAU Circ., 3147, 1

Krisciunas, K., Sinton, W., Tholen, K., et al. 1987, PASP, 99, 887

Lacerda, P., Jewitt, D., \& Peixinho, N. 2008, AJ, 135, 1749

Landolt, A. U. 1992, AJ, 104, 340

Lazzaro, D., Florczak, M. A., Angeli, C. A., et al. 1997, Planet. Space Sci., 45, 1607

Lehmann, E. L. 1993, J. Am. Stat. Assoc., 88, 1242

Leinhardt, Z. M., Stewart, S. T., \& Schultz, P. H. 2008, Physical Effects of Collisions in the Kuiper Belt, eds. M. A. Barucci, H. Boehnhardt, D. P. Cruikshank, A., Morbidelli, \& R. Dotson, 195

Levison, H. F., \& Duncan, M. J. 1997, Icarus, 127, 13

Luu, J., \& Jewitt, D. 1996, AJ, 112, 2310

Lykawka, P. S., \& Mukai, T. 2007, Icarus, 189, 213

Maechler, M. 2011, Diptest: Hartigan's dip test statistic for unimodality - corrected code, $r$ package version $0.75-1$

Merk, R., \& Prialnik, D. 2006, Icarus, 183, 283

Merlin, F., Alvarez-Candal, A., Delsanti, A., et al. 2009, AJ, 137, 315

Mommert, M., Harris, A. W., Kiss, C., et al. 2012, A\&A, 541, A93

Pan, M., \& Sari, R. 2005, Icarus, 173, 342

Peixinho, N., Lacerda, P., Ortiz, J. L., et al. 2001, A\&A, 371, 753

Peixinho, N., Doressoundiram, A., Delsanti, A., et al. 2003, A\&A, 410, L29

Peixinho, N., Boehnhardt, H., Belskaya, I., et al. 2004, Icarus, 170, 153

Pinilla-Alonso, N., Brunetto, R., Licandro, J., et al. 2009, A\&A, 496, 547

R Development Core Team 2011, R: A Language and Environment for Statistical Computing, R Foundation for Statistical Computing, Vienna, Austria

Rabinowitz, D. L., Barkume, K., Brown, M. E., et al. 2006, ApJ, 639, 1238

Rabinowitz, D. L., Schaefer, B. E., \& Tourtellotte, S. W. 2007, AJ, 133, 26

Rabinowitz, D. L., Schaefer, B. E., Schaefer, M., \& Tourtellotte, S. W. 2008, AJ, 136, 1502

Romanishin, W., Tegler, S. C., Levine, J., \& Butler, N. 1997, AJ, 113, 1893

Romanishin, W., Tegler, S. C., \& Consolmagno, G. J. 2010, AJ, 140, 29

Romon-Martin, J., Barucci, M. A., de Bergh, C., et al. 2002, Icarus, 160, 59

Romon-Martin, J., Delahodde, C., Barucci, M. A., de Bergh, C., \& Peixinho, N. 2003, A\&A, 400, 369

Santos-Sanz, P., Ortiz, J. L., Barrera, L., \& Boehnhardt, H. 2009, A\&A, 494, 693

Santos-Sanz, P., Lellouch, E., Fornasier, S., et al. 2012, A\&A, 541, A92 
N. Peixinho et al.: The bimodal colors of Centaurs and small Kuiper belt objects

Schaller, E. L., \& Brown, M. E. 2007, ApJ, 659, L61

Schaller, E. L., \& Brown, M. E. 2008, ApJ, 684, L107

Sheppard, S. S. 2010, AJ, 139, 1394

Sheppard, S. S., \& Trujillo, C. A. 2006, Science, 313, 511

Snodgrass, C., Carry, B., Dumas, C., \& Hainaut, O. 2010, A\&A, 511, A72

Stansberry, J., Grundy, W., Brown, M., et al. 2008, Physical Properties of Kuiper Belt and Centaur Objects: Constraints from the Spitzer Space Telescope, eds. M. A. Barucci, H. Boehnhardt, D. P. Cruikshank, A. Morbidelli, \& R. Dotson, 161

Tegler, S. C., \& Romanishin, W. 1997, Icarus, 126, 212

Tegler, S. C., \& Romanishin, W. 1998, Nature, 392, 49

Tegler, S. C., \& Romanishin, W. 2000, Nature, 407, 979
Tegler, S. C., \& Romanishin, W. 2003, Icarus, 161, 181

Tegler, S. C., Romanishin, W., \& Consolmagno, G. J. 2003, ApJ, 599, L49

Tegler, S. C., Bauer, J. M., Romanishin, W., \& Peixinho, N. 2008, Colors of Centaurs, eds. M. A. Barucci, H. Boehnhardt, D. P. Cruikshank, A. Morbidelli, \& R. Dotson, 105

Thébault, P., \& Doressoundiram, A. 2003, Icarus, 162, 27

Thompson, W. R., Murray, B. G. J. P. T., Khare, B. N., \& Sagan, C. 1987, J. Geophys. Res., 92, 14933

Trujillo, C. A., \& Brown, M. E. 2002, ApJ, 566, L125

Vilenius, E., Kiss, C., Mommert, M., et al. 2012, A\&A, 541, A94

Volk, K., \& Malhotra, R. 2008, ApJ, 687, 714

Yu, Q., \& Tremaine, S. 1999, ApJ, 118, 1873

Pages 8 to 12 are available in the electronic edition of the journal at http://www . aanda. org 
Table 3. Compilation of absolute magnitude $H_{R}(\alpha), B-R$ colors, and spectral features used in this work.

\begin{tabular}{|c|c|c|c|c|c|}
\hline Object & Dynamical class & $H_{R}(\alpha)$ & $\overline{B-R}$ & Spectral features & References \\
\hline (2060) Chiron & Centaur & $6.287 \pm 0.022$ & $1.010 \pm 0.044$ & Water ice & $1,2,3, \mathrm{a}$ \\
\hline (5145) Pholus & Centaur & $7.198 \pm 0.056$ & $1.970 \pm 0.108$ & Methanol & $4, b$ \\
\hline (7066) Nessus & Centaur & $9.020 \pm 0.068$ & $1.847 \pm 0.165$ & & 1 \\
\hline (8405) Asbolus & Centaur & $9.257 \pm 0.120$ & $1.228 \pm 0.057$ & Water ice & $4,5, \mathrm{c}$ \\
\hline (10199) Chariklo & Centaur & $6.569 \pm 0.015$ & $1.299 \pm 0.065$ & Water ice & $6,7, d$ \\
\hline (10370) Hylonome & Centaur & $9.250 \pm 0.131$ & $1.153 \pm 0.081$ & & $1,6,8$ \\
\hline (15760) $1992 \mathrm{QB}_{1}$ & Cold classical & $6.867 \pm 0.121$ & $1.670 \pm 0.145$ & & $1,7,9$ \\
\hline (15788) $1993 \mathrm{SB}$ & Plutino & $8.032 \pm 0.122$ & $1.276 \pm 0.100$ & & $7,9,10$ \\
\hline (15789) $1993 \mathrm{SC}$ & Plutino & $6.722 \pm 0.074$ & $1.720 \pm 0.140$ & Methane & $1,7,11,12, \mathrm{e}$ \\
\hline (15810) $1994 \mathrm{JR}_{1}$ & Plutino & $6.867 \pm 0.077$ & $1.610 \pm 0.216$ & & 13 \\
\hline (15820) 1994 ТВ & Plutino & $7.527 \pm 0.091$ & $1.759 \pm 0.155$ & & $1,7,10,11,13$ \\
\hline (15874) $1996 \mathrm{TL}_{66}$ & Scattered disk object & $5.131 \pm 0.144$ & $1.113 \pm 0.070$ & & $6,7,12,13,14$ \\
\hline (15875) $1996 \mathrm{TP}_{66}$ & Plutino & $6.953 \pm 0.071$ & $1.678 \pm 0.123$ & & $6,7,12,13,14,15$ \\
\hline (15883) $1997 \mathrm{CR}_{29}$ & Scattered disk object & $7.076 \pm 0.135$ & $1.260 \pm 0.128$ & & 7,16 \\
\hline (16684) $1994 \mathrm{JQ}_{1}$ & Cold classical & $6.618 \pm 0.117$ & $1.738 \pm 0.120$ & & $17,18,19$ \\
\hline (19255) $1994 \mathrm{VK}_{8}$ & Cold classical & $7.016 \pm 0.163$ & $1.680 \pm 0.067$ & & 9 \\
\hline (19299) $1996 \mathrm{SZ}_{4}$ & Plutino & $8.184 \pm 0.159$ & $1.299 \pm 0.102$ & & $7,9,18$ \\
\hline (19308) $1996 \mathrm{TO}_{66}$ & Resonant (19:11) & $4.530 \pm 0.044$ & $1.056 \pm 0.210$ & Water ice & $6,7,12,13,14,20,21, f$ \\
\hline (19521) Chaos & Hot classical & $4.442 \pm 0.069$ & $1.558 \pm 0.062$ & & $8,9,10,22$ \\
\hline (20000) Varuna & Hot classical & $3.345 \pm 0.059$ & $1.530 \pm 0.036$ & Water ice & $8, \mathrm{~g}$ \\
\hline (20108) $1995 \mathrm{QZ}_{9}$ & Plutino & $7.889 \pm 0.399$ & $1.400 \pm 0.050$ & & 9 , This work \\
\hline (24835) $1995 \mathrm{SM}_{55}$ & Hot classical & $4.352 \pm 0.040$ & $1.018 \pm 0.052$ & Water ice & $8,10,14,23, \mathrm{~g}$ \\
\hline (24952) $1997 \mathrm{QJ}_{4}$ & Plutino & $7.389 \pm 0.114$ & $1.104 \pm 0.104$ & & 7,18 \\
\hline (24978) $1998 \mathrm{HJ}_{151}$ & Cold classical & $7.008 \pm 0.050$ & $1.820 \pm 0.042$ & & 19 \\
\hline (26181) $1996 \mathrm{GQ}_{21}$ & Resonant (11:2) & $4.467 \pm 0.090$ & $1.693 \pm 0.079$ & Methanol & $18,24, \mathrm{~g}$ \\
\hline (26308) $1998 \mathrm{SM}_{165}$ & Resonant (2:1) & $5.757 \pm 0.119$ & $1.620 \pm 0.105$ & & $9,10,15$ \\
\hline (26375) $1999 \mathrm{DE}_{9}$ & Resonant (5:2) & $4.810 \pm 0.046$ & $1.536 \pm 0.056$ & Featureless & $7,8,25, \mathrm{~h}$ \\
\hline (28978) Ixion & Scattered disk object & $3.366 \pm 0.038$ & $1.634 \pm 0.035$ & Water ice & $8, \mathrm{~h}$ \\
\hline (29981) $1999 \mathrm{TD}_{10}$ & Scattered disk object & $8.698 \pm 0.038$ & $1.230 \pm 0.028$ & Water ice & $8, \mathrm{~g}$ \\
\hline (31824) Elatus & Centaur & $10.439 \pm 0.107$ & $1.672 \pm 0.071$ & Water ice & $8,10,26, \mathrm{~g}$ \\
\hline (32532) Thereus & Centaur & $9.365 \pm 0.038$ & $1.190 \pm 0.032$ & Water ice & $25, \mathrm{~h}$ \\
\hline (32929) $1995 \mathrm{QY}_{9}$ & Plutino & $7.489 \pm 0.126$ & $1.160 \pm 0.150$ & & 1,13 \\
\hline (33001) $1997 \mathrm{CU}_{29}$ & Cold classical & $6.173 \pm 0.078$ & $1.804 \pm 0.115$ & & $7,16,22,27$ \\
\hline (33128) $1998 \mathrm{BU}_{48}$ & Scattered disk object & $6.889 \pm 0.127$ & $1.692 \pm 0.089$ & & 8,10 \\
\hline (33340) $1998 \mathrm{VG}_{44}$ & Plutino & $6.292 \pm 0.077$ & $1.511 \pm 0.055$ & & $8,14,16,24$ \\
\hline (35671) $1998 \mathrm{SN}_{165}$ & Scattered disk object & $5.431 \pm 0.068$ & $1.123 \pm 0.082$ & & $7,10,16$ \\
\hline (38083) Rhadamanthus & Scattered disk object & $7.432 \pm 0.063$ & $1.177 \pm 0.109$ & & 18 \\
\hline (38084) $1999 \mathrm{HB}_{12}$ & Resonant (5:2) & $6.718 \pm 0.050$ & $1.409 \pm 0.049$ & & $16,25,27,28$ \\
\hline (38628) Huya & Plutino & $4.674 \pm 0.099$ & $1.539 \pm 0.062$ & Featureless & $29,7,16,18, \mathrm{~g}$ \\
\hline (40314) $1999 \mathrm{KR}_{16}$ & Scattered disk object & $5.527 \pm 0.039$ & $1.872 \pm 0.068$ & & $7,18,27$ \\
\hline (42301) $2001 \mathrm{UR}_{163}$ & Resonant (9:4) & $3.812 \pm 0.109$ & $2.190 \pm 0.130$ & Featureless & $15,30,31, \mathrm{~g}$ \\
\hline (42355) Typhon & Scattered disk object & $7.358 \pm 0.076$ & $1.292 \pm 0.071$ & Water ice & $25,28, \mathrm{~h}$ \\
\hline (44594) $1999 \mathrm{OX}_{3}$ & Scattered disk object & $6.835 \pm 0.078$ & $1.839 \pm 0.087$ & Water ice & $8,9,10,15,21,30, \mathrm{i}$ \\
\hline (47171) $1999 \mathrm{TC}_{36}$ & Plutino & $4.851 \pm 0.054$ & $1.740 \pm 0.049$ & Water ice & $10,16,25,32, \mathrm{~h}$ \\
\hline (47932) $2000 \mathrm{GN}_{171}$ & Plutino & $5.666 \pm 0.090$ & $1.559 \pm 0.066$ & Featureless & $18,24, \mathrm{~h}$ \\
\hline (48639) $1995 \mathrm{TL}_{8}$ & Detached KBO & $4.667 \pm 0.091$ & $1.693 \pm 0.217$ & & $8,10,21$ \\
\hline (49036) Pelion & Centaur & $10.157 \pm 0.112$ & $1.248 \pm 0.096$ & & 9,18 \\
\hline (50000) Quaoar & Hot classical & $2.220 \pm 0.029$ & $1.588 \pm 0.021$ & Methane & $25,33, \mathrm{~h}$ \\
\hline (52747) $1998 \mathrm{HM}_{151}$ & Cold classical & $7.417 \pm 0.100$ & $1.550 \pm 0.103$ & & 19 \\
\hline (52872) Okyrhoe & Centaur & $10.775 \pm 0.078$ & $1.237 \pm 0.086$ & Water ice & $10,16,32, \mathrm{~g}$ \\
\hline (52975) Cyllarus & Centaur & $8.634 \pm 0.101$ & $1.803 \pm 0.102$ & & $8,10,14,25$ \\
\hline (53311) Deucalion & Cold classical & $6.662 \pm 0.060$ & $2.030 \pm 0.160$ & & 27 \\
\hline (54598) Bienor & Centaur & $7.727 \pm 0.077$ & $1.158 \pm 0.075$ & Methanol & $8,10,15, \mathrm{~h}$ \\
\hline (55565) $2002 \mathrm{AW}_{197}$ & Hot classical & $3.156 \pm 0.059$ & $1.498 \pm 0.044$ & Featureless & $24,33,34, \mathrm{~h}$ \\
\hline (55576) Amycus & Centaur & $7.789 \pm 0.042$ & $1.814 \pm 0.044$ & Water ic & $24,28,33,34, \mathrm{i}$ \\
\hline (55636) $2002 \mathrm{TX}_{300}$ & Hot classical & $3.296 \pm 0.047$ & $1.010 \pm 0.028$ & Water ice & $25,30, \mathrm{q}$ \\
\hline (55637) $2002 \mathrm{UX}_{25}$ & Scattered disk object & $3.486 \pm 0.084$ & $1.502 \pm 0.052$ & Water ice & $24,31, \mathrm{~g}$ \\
\hline (55638) $2002 \mathrm{VE}_{95}$ & Plutino & $5.143 \pm 0.062$ & $1.790 \pm 0.040$ & Methanol & $24, \mathrm{~g}$ \\
\hline (58534) Logos & Cold classical & $6.759 \pm 0.181$ & $1.653 \pm 0.150$ & & 7,22 \\
\hline (59358) $1999 \mathrm{CL}_{158}$ & Scattered disk object & $6.653 \pm 0.090$ & $1.190 \pm 0.072$ & & 8 \\
\hline
\end{tabular}


N. Peixinho et al.: The bimodal colors of Centaurs and small Kuiper belt objects

Table 3. continued.

\begin{tabular}{|c|c|c|c|c|c|}
\hline Object & Dynamical class & $\overline{H_{R}(\alpha)}$ & $\overline{B-R}$ & Spectral features & References \\
\hline (60454) $2000 \mathrm{CH}_{105}$ & Cold classical & $6.363 \pm 0.077$ & $1.699 \pm 0.083$ & & 28 \\
\hline (60458) $2000 \mathrm{CM}_{114}$ & Scattered disk object & $6.954 \pm 0.044$ & $1.240 \pm 0.040$ & & 25 \\
\hline (60558) Echeclus & Centaur & $9.669 \pm 0.090$ & $1.376 \pm 0.072$ & & 18,24 \\
\hline (60608) $2000 \mathrm{EE}_{173}$ & Scattered disk object & $8.028 \pm 0.107$ & $1.164 \pm 0.032$ & & 18,25 \\
\hline (60620) $2000 \mathrm{FD}_{8}$ & Resonant (7:4) & $6.344 \pm 0.061$ & $1.806 \pm 0.113$ & & 18,28 \\
\hline (60621) $2000 \mathrm{FE}_{8}$ & Resonant (5:2) & $6.510 \pm 0.062$ & $1.230 \pm 0.027$ & & 8,25 \\
\hline (63252) $2001 \mathrm{BL}_{41}$ & Centaur & $11.273 \pm 0.065$ & $1.199 \pm 0.045$ & & 25,28 \\
\hline (65489) Ceto & Scattered disk object & $6.205 \pm 0.060$ & $1.420 \pm 0.040$ & Water ice & $25, \mathrm{~g}$ \\
\hline (66452) $1999 \mathrm{OF}_{4}$ & Cold classical & $6.255 \pm 0.090$ & $1.830 \pm 0.095$ & & 28 \\
\hline (66652) Borasisi & Cold classical & $5.420 \pm 0.051$ & $1.610 \pm 0.050$ & & 16,35 \\
\hline (69986) $1998 \mathrm{WW}_{24}$ & Plutino & $7.964 \pm 0.096$ & $1.235 \pm 0.152$ & & 8,28 \\
\hline (69988) $1998 \mathrm{WA}_{31}$ & Resonant (5:2) & $7.303 \pm 0.149$ & $1.412 \pm 0.127$ & & 28 \\
\hline (69990) $1998 \mathrm{WU}_{31}$ & Plutino & $7.988 \pm 0.200$ & $1.225 \pm 0.086$ & & 28 \\
\hline (73480) $2002 \mathrm{PN}_{34}$ & Scattered disk object & $8.487 \pm 0.046$ & $1.280 \pm 0.020$ & Water ice & $25, \mathrm{j}$ \\
\hline (79360) $1997 \mathrm{CS}_{29}$ & Cold classical & $5.068 \pm 0.085$ & $1.746 \pm 0.077$ & Featureless & $6,7,14,22, \mathrm{k}$ \\
\hline (79978) $1999 \mathrm{CC}_{158}$ & Resonant (12:5) & $5.409 \pm 0.091$ & $1.566 \pm 0.100$ & & $8,10,24$ \\
\hline (79983) $1999 \mathrm{DF}_{9}$ & Hot classical & $5.797 \pm 0.110$ & $1.630 \pm 0.078$ & & 8 \\
\hline (80806) $2000 \mathrm{CM}_{105}$ & Cold classical & $6.302 \pm 0.030$ & $1.980 \pm 0.230$ & & 27 \\
\hline (82075) $2000 \mathrm{YW}_{134}$ & Resonant (8:3) & $4.429 \pm 0.064$ & $1.417 \pm 0.077$ & & $21,25,28,30,31$ \\
\hline (82155) $2001 \mathrm{FZ}_{173}$ & Scattered disk object & $5.811 \pm 0.027$ & $1.418 \pm 0.030$ & & 25,28 \\
\hline (82158) $2001 \mathrm{FP}_{185}$ & Scattered disk object & $5.940 \pm 0.053$ & $1.402 \pm 0.055$ & & 25,30 \\
\hline (83982) Crantor & Centaur & $8.693 \pm 0.057$ & $1.864 \pm 0.044$ & Methanol & $25,28,33,34, \mathrm{~h}$ \\
\hline (84522) $2002 \mathrm{TC}_{302}$ & Scattered or detached KBO & $3.682 \pm 0.067$ & $1.741 \pm 0.048$ & Water ice & $21,24,31, \mathrm{~g}$ \\
\hline (84719) $2002 \mathrm{VR}_{128}$ & Plutino & $5.005 \pm 0.040$ & $1.540 \pm 0.040$ & & 24 \\
\hline (84922) $2003 \mathrm{VS}_{2}$ & Plutino & $3.794 \pm 0.070$ & $1.520 \pm 0.030$ & Water ice & $24, \mathrm{~g}$ \\
\hline (85633) $1998 \mathrm{KR}_{65}$ & Cold classical & $6.599 \pm 0.073$ & $1.727 \pm 0.144$ & & 18,19 \\
\hline (86047) $1999 \mathrm{OY}_{3}$ & Scattered disk object & $6.293 \pm 0.055$ & $1.055 \pm 0.050$ & & $8,9,18$ \\
\hline (86177) $1999 \mathrm{RY}_{215}$ & Scattered disk object & $6.736 \pm 0.114$ & $1.151 \pm 0.183$ & & 16,18 \\
\hline (87269) $2000 \mathrm{OO}_{67}$ & Scattered disk object & $9.057 \pm 0.170$ & $1.702 \pm 0.092$ & & 21,25 \\
\hline$(87555) 2000 \mathrm{QB}_{243}$ & Scattered disk object & $8.439 \pm 0.119$ & $1.088 \pm 0.094$ & & 15,28 \\
\hline (88269) $2001 \mathrm{KF}_{77}$ & Centaur & $10.038 \pm 0.020$ & $1.810 \pm 0.040$ & & 25 \\
\hline (90377) Sedna & Detached KBO & $1.120 \pm 0.088$ & $1.874 \pm 0.115$ & Methane & $21,24,36,1$ \\
\hline (90482) Orcus & Scattered disk object & $1.991 \pm 0.054$ & $1.042 \pm 0.037$ & Methane & $24,36, \mathrm{~m}$ \\
\hline (90568) $2004 \mathrm{GV}_{9}$ & Hot classical & $3.786 \pm 0.080$ & $1.470 \pm 0.040$ & Featureless & $24, \mathrm{~h}$ \\
\hline (91133) $1998 \mathrm{HK}_{151}$ & Plutino & $6.937 \pm 0.076$ & $1.240 \pm 0.064$ & & 8,16 \\
\hline (91205) $1998 \mathrm{US}_{43}$ & Plutino & $7.852 \pm 0.050$ & $1.185 \pm 0.102$ & & 28 \\
\hline (91554) $1999 \mathrm{RZ}_{215}$ & Scattered disk object & $8.072 \pm 0.079$ & $1.346 \pm 0.132$ & & 18 \\
\hline (95626) $2002 \mathrm{GZ}_{32}$ & Centaur & $6.603 \pm 0.131$ & $1.199 \pm 0.075$ & & $25,30,33$ \\
\hline (118228) $1996 \mathrm{TQ}_{66}$ & Plutino & $7.245 \pm 0.195$ & $1.881 \pm 0.144$ & & 6,7 \\
\hline (118378) $1999 \mathrm{HT}_{11}$ & Resonant (7:4) & $6.906 \pm 0.040$ & $1.830 \pm 0.100$ & & 27 \\
\hline (118379) $1999 \mathrm{HC}_{12}$ & Scattered disk object & $7.611 \pm 0.170$ & $1.384 \pm 0.214$ & & 18 \\
\hline (118702) $2000 \mathrm{OM}_{67}$ & Scattered or detached KBO & $7.075 \pm 0.036$ & $1.290 \pm 0.040$ & & 21 \\
\hline (119068) $2001 \mathrm{KC}_{77}$ & Resonant (5:2) & $6.822 \pm 0.030$ & $1.470 \pm 0.010$ & & 25 \\
\hline (119070) $2001 \mathrm{KP}_{77}$ & Resonant (7:4) & $6.873 \pm 0.305$ & $1.720 \pm 0.319$ & & 28,30 \\
\hline (119315) $2001 \mathrm{SQ}_{73}$ & Centaur & $8.857 \pm 0.069$ & $1.130 \pm 0.020$ & & 25,31 \\
\hline (119473) $2001 \mathrm{UO}_{18}$ & Plutino & $7.804 \pm 0.506$ & $2.079 \pm 0.376$ & & 30 \\
\hline (119878) $2002 \mathrm{CY}_{224}$ & Resonant (12:5) & $5.871 \pm 0.056$ & $1.680 \pm 0.100$ & & 31 \\
\hline (119951) $2002 \mathrm{KX}_{14}$ & Scattered disk object & $4.349 \pm 0.124$ & $1.660 \pm 0.040$ & Featureless & $24,37, \mathrm{~h}$ \\
\hline (120061) $2003 \mathrm{CO}_{1}$ & Centaur & $9.134 \pm 0.140$ & $1.240 \pm 0.040$ & & 25,27 \\
\hline (120132) $2003 \mathrm{FY}_{128}$ & Scattered disk object & $4.486 \pm 0.053$ & $1.650 \pm 0.020$ & Water ice & $21, \mathrm{~g}$ \\
\hline (120181) $2003 \mathrm{UR}_{292}$ & Scattered disk object & $7.093 \pm 0.100$ & $1.690 \pm 0.080$ & & 24 \\
\hline (120216) $2004 \mathrm{EW}_{95}$ & Plutino & $6.309 \pm 0.050$ & $1.080 \pm 0.030$ & & 24 \\
\hline (121725) $1999 \mathrm{XX}_{143}$ & Centaur & $8.586 \pm 0.096$ & $1.734 \pm 0.145$ & & 8,28 \\
\hline (126619) $2002 \mathrm{CX}_{154}$ & Scattered or detached KBO & $7.178 \pm 0.075$ & $1.470 \pm 0.128$ & & 31 \\
\hline (127546) $2002 \mathrm{XU}_{93}$ & Scattered disk object & $7.942 \pm 0.019$ & $1.200 \pm 0.020$ & & 21 \\
\hline (129772) $1999 \mathrm{HR}_{11}$ & Resonant (7:4) & $7.172 \pm 0.150$ & $1.450 \pm 0.156$ & & 16 \\
\hline (130391) $2000 \mathrm{JG}_{81}$ & Resonant (2:1) & $7.748 \pm 0.056$ & $1.417 \pm 0.060$ & & This work \\
\hline (134860) $2000 \mathrm{OJ}_{67}$ & Cold classical & $6.001 \pm 0.120$ & $1.720 \pm 0.078$ & & 8 \\
\hline (135182) $2001 \mathrm{QT}_{322}$ & Scattered disk object & $7.752 \pm 0.320$ & $1.240 \pm 0.060$ & & 37 \\
\hline (136108) Haumea & Resonant(12:7) & $0.205 \pm 0.011$ & $0.973 \pm 0.024$ & Water ice & $38,39, \mathrm{n}$ \\
\hline (136120) $2003 \mathrm{LG}_{7}$ & Resonant (3:1) & $8.322 \pm 0.049$ & $1.271 \pm 0.091$ & & This work \\
\hline
\end{tabular}


Table 3. continued.

\begin{tabular}{|c|c|c|c|c|c|}
\hline Object & Dynamical class & $\overline{H_{R}(\alpha)}$ & $\overline{B-R}$ & Spectral features & References \\
\hline (136199) Eris & Scattered or detached KBO & $-1.511 \pm 0.033$ & $1.207 \pm 0.088$ & Methane & $24,36, \mathrm{o}$ \\
\hline (136204) $2003 \mathrm{WL}_{7}$ & Centaur & $8.670 \pm 0.070$ & $1.230 \pm 0.040$ & & 24 \\
\hline (136472) Makemake & Hot classical & $-0.317 \pm 0.024$ & $1.332 \pm 0.029$ & Methane & $36, p$ \\
\hline (137294) $1999 \mathrm{RE}_{215}$ & Cold classical & $6.091 \pm 0.073$ & $1.700 \pm 0.148$ & & 18 \\
\hline (137295) $1999 \mathrm{RB}_{216}$ & Resonant $(2: 1)$ & $7.668 \pm 0.096$ & $1.419 \pm 0.142$ & & 18 \\
\hline (138537) $2000 \mathrm{OK}_{67}$ & Cold classical & $6.093 \pm 0.083$ & $1.540 \pm 0.094$ & & 8 \\
\hline (144897) $2004 \mathrm{UX}_{10}$ & Hot classical & $4.216 \pm 0.087$ & $1.530 \pm 0.020$ & Methanol & $37, \mathrm{i}$ \\
\hline (145480) $2005 \mathrm{~TB}_{190}$ & Detached KBO & $3.949 \pm 0.085$ & $1.540 \pm 0.030$ & & 21 \\
\hline (148209) $2000 \mathrm{CR}_{105}$ & Detached KBO & $6.191 \pm 0.073$ & $1.273 \pm 0.068$ & & 21,25 \\
\hline (148780) Altjira & Hot classical & $5.885 \pm 0.320$ & $1.640 \pm 0.170$ & & 30 \\
\hline (149560) $2003 \mathrm{QZ}_{91}$ & Scattered disk object & $8.302 \pm 0.028$ & $1.305 \pm 0.048$ & & This work \\
\hline (168703) $2000 \mathrm{GP}_{183}$ & Scattered disk object & $5.795 \pm 0.061$ & $1.160 \pm 0.057$ & & 8 \\
\hline (181708) $1993 \mathrm{FW}$ & Hot classical & $6.572 \pm 0.105$ & $1.625 \pm 0.110$ & & $1,17,19,22$ \\
\hline (181855) $1998 \mathrm{WT}_{31}$ & Hot classical & $7.443 \pm 0.079$ & $1.247 \pm 0.140$ & & 28,40 \\
\hline (181867) $1999 \mathrm{CV}_{118}$ & Resonant $(7: 3)$ ? & $7.067 \pm 0.163$ & $2.130 \pm 0.090$ & & 27 \\
\hline (181868) $1999 \mathrm{CG}_{119}$ & Scattered disk object & $7.004 \pm 0.040$ & $1.530 \pm 0.080$ & & 27 \\
\hline (181871) $1999 \mathrm{CO}_{153}$ & Cold classical & $6.607 \pm 0.030$ & $1.940 \pm 0.090$ & & 27 \\
\hline (181874) $1999 \mathrm{HW}_{11}$ & Scattered or detached KBO & $6.706 \pm 0.062$ & $1.323 \pm 0.043$ & & 21,27 \\
\hline (182397) $2001 \mathrm{QW}_{297}$ & Resonant (9:4) & $6.660 \pm 0.064$ & $1.600 \pm 0.070$ & & 21 \\
\hline (182934) $2002 \mathrm{GJ}_{32}$ & Hot classical & $5.469 \pm 0.187$ & $1.678 \pm 0.261$ & & 30,31 \\
\hline 1993 RO & Plutino & $8.492 \pm 0.113$ & $1.385 \pm 0.154$ & & 1,9 \\
\hline $1994 \mathrm{EV}_{3}$ & Cold classical & $7.110 \pm 0.072$ & $1.732 \pm 0.167$ & & $1,18,27$ \\
\hline 1994 TA & Centaur & $11.421 \pm 0.126$ & $1.930 \pm 0.155$ & & 9,7 \\
\hline $1995 \mathrm{HM}_{5}$ & Plutino & $7.849 \pm 0.109$ & $1.010 \pm 0.192$ & & 6,22 \\
\hline $1995 \mathrm{WY}_{2}$ & Cold classical & $6.864 \pm 0.110$ & $1.655 \pm 0.278$ & & 1,7 \\
\hline $1996 \mathrm{RQ}_{20}$ & Hot classical & $6.903 \pm 0.092$ & $1.523 \pm 0.156$ & & 7,10 \\
\hline $1996 \mathrm{RR}_{20}$ & Plutino & $6.622 \pm 0.143$ & $1.868 \pm 0.130$ & & $7,9,18$ \\
\hline $1996 \mathrm{TK}_{66}$ & Cold classical & $6.190 \pm 0.116$ & $1.666 \pm 0.088$ & & $7,8,9$ \\
\hline $1996 \mathrm{TS}_{66}$ & Hot classical & $5.947 \pm 0.130$ & $1.665 \pm 0.157$ & & $6,7,12$ \\
\hline $1997 \mathrm{CV}_{29}$ & Hot classical & $7.154 \pm 0.030$ & $1.860 \pm 0.022$ & & 19 \\
\hline $1997 \mathrm{QH}_{4}$ & Hot classical & $6.996 \pm 0.136$ & $1.731 \pm 0.168$ & & $7,9,10,18$ \\
\hline $1997 \mathrm{RT}_{5}$ & Hot classical & $7.117 \pm 0.140$ & $1.549 \pm 0.162$ & & 18 \\
\hline $1997 \mathrm{SZ}_{10}$ & Resonant (2:1) & $8.100 \pm 0.104$ & $1.790 \pm 0.085$ & & 9 \\
\hline $1998 \mathrm{FS}_{144}$ & Hot classical & $6.717 \pm 0.105$ & $1.516 \pm 0.057$ & & 19,22 \\
\hline $1998 \mathrm{HL}_{151}$ & Hot classical & $8.120 \pm 0.149$ & $1.190 \pm 0.284$ & & 27,40 \\
\hline $1998 \mathrm{KG}_{62}$ & Cold classical & $6.125 \pm 0.110$ & $1.602 \pm 0.158$ & & 16,18 \\
\hline $1998 \mathrm{KS}_{65}$ & Cold classical & $7.166 \pm 0.040$ & $1.730 \pm 0.045$ & & 19 \\
\hline $1998 \mathrm{UR}_{43}$ & Plutino & $8.083 \pm 0.132$ & $1.390 \pm 0.113$ & & 10 \\
\hline $1998 \mathrm{WS}_{31}$ & Plutino & $7.952 \pm 0.186$ & $1.315 \pm 0.075$ & & 28 \\
\hline $1998 \mathrm{WV}_{24}$ & Cold classical & $7.126 \pm 0.067$ & $1.270 \pm 0.032$ & & 9 \\
\hline $1998 \mathrm{WV}_{31}$ & Plutino & $7.627 \pm 0.069$ & $1.349 \pm 0.096$ & & 10,28 \\
\hline $1998 \mathrm{WX}_{24}$ & Cold classical & $6.241 \pm 0.099$ & $1.790 \pm 0.071$ & & 9 \\
\hline $1998 \mathrm{WZ}_{31}$ & Plutino & $8.044 \pm 0.102$ & $1.263 \pm 0.089$ & & 28 \\
\hline $1998 X_{95}$ & Scattered or detached $\mathrm{KBO}$ & $6.438 \pm 0.143$ & $1.580 \pm 0.212$ & & 14 \\
\hline $1999 \mathrm{CB}_{119}$ & Hot classical & $6.740 \pm 0.050$ & $1.926 \pm 0.095$ & & 28 \\
\hline $1999 \mathrm{CD}_{158}$ & Resonant $(7: 4)$ & $4.837 \pm 0.111$ & $1.384 \pm 0.116$ & & $8,10,40$ \\
\hline $1999 \mathrm{CF}_{119}$ & Scattered or detached $\mathrm{KBO}$ & $6.982 \pm 0.084$ & $1.424 \pm 0.072$ & & 27,25 \\
\hline $1999 \mathrm{CJ}_{119}$ & Cold classical & $6.695 \pm 0.210$ & $2.070 \pm 0.220$ & & 27 \\
\hline $1999 \mathrm{CM}_{119}$ & Cold classical & $7.356 \pm 0.060$ & $1.780 \pm 0.170$ & & 27 \\
\hline $1999 C_{133}$ & Hot classical & $6.682 \pm 0.050$ & $1.350 \pm 0.070$ & & 27 \\
\hline $1999 \mathrm{CX}_{131}$ & Resonant (5:3) & $6.914 \pm 0.087$ & $1.637 \pm 0.118$ & & 28 \\
\hline $1999 \mathrm{GS}_{46}$ & Hot classical & $6.230 \pm 0.020$ & $1.760 \pm 0.070$ & & 27 \\
\hline $1999 \mathrm{HS}_{11}$ & Cold classical & $6.344 \pm 0.081$ & $1.845 \pm 0.099$ & & $16,19,28,35$ \\
\hline $1999 \mathrm{HV}_{11}$ & Cold classical & $7.003 \pm 0.050$ & $1.700 \pm 0.063$ & & 19 \\
\hline $1999 \mathrm{JD}_{132}$ & Hot classical & $5.983 \pm 0.020$ & $1.590 \pm 0.090$ & & 27 \\
\hline $1999 \mathrm{OE}_{4}$ & Cold classical & $6.887 \pm 0.193$ & $1.832 \pm 0.147$ & & 28 \\
\hline $1999 \mathrm{OJ}_{4}$ & Cold classical & $6.899 \pm 0.060$ & $1.675 \pm 0.077$ & & 28 \\
\hline $1999 \mathrm{OM}_{4}$ & Cold classical & $7.521 \pm 0.100$ & $1.739 \pm 0.170$ & & 18 \\
\hline $1999 \mathrm{RJ}_{215}$ & Scattered disk object & $7.881 \pm 0.103$ & $1.221 \pm 0.175$ & & 18 \\
\hline $1999 \mathrm{RX}_{214}$ & Cold classical & $6.385 \pm 0.050$ & $1.647 \pm 0.070$ & & 28 \\
\hline $1999 \mathrm{RY}_{214}$ & Hot classical & $7.006 \pm 0.040$ & $1.258 \pm 0.085$ & & 28 \\
\hline $1999 \mathrm{TR}_{11}$ & Plutino & $8.063 \pm 0.140$ & $1.770 \pm 0.106$ & & 9 \\
\hline $2000 \mathrm{AF}_{255}$ & Scattered disk object & $5.682 \pm 0.030$ & $1.780 \pm 0.060$ & & 27 \\
\hline $2000 \mathrm{CG}_{105}$ & Hot classical & $6.469 \pm 0.293$ & $1.170 \pm 0.170$ & & 27,40 \\
\hline $2000 \mathrm{CJ}_{105}$ & Hot classical & $5.687 \pm 0.066$ & $1.760 \pm 0.106$ & & 31 \\
\hline $2000 \mathrm{CL}_{104}$ & Cold classical & $6.394 \pm 0.086$ & $1.851 \pm 0.192$ & & 18 \\
\hline $2000 \mathrm{CL}_{105}$ & Cold classical & $6.761 \pm 0.060$ & $1.520 \pm 0.090$ & & 27 \\
\hline
\end{tabular}


N. Peixinho et al.: The bimodal colors of Centaurs and small Kuiper belt objects

Table 3. continued.

\begin{tabular}{|c|c|c|c|c|c|}
\hline Object & Dynamical class & $\overline{H_{R}(\alpha)}$ & $B-R$ & Spectral features & References \\
\hline $2000 \mathrm{CN}_{105}$ & Cold classical & $5.286 \pm 0.160$ & $1.720 \pm 0.128$ & & 31 \\
\hline $2000 \mathrm{CO}_{105}$ & Hot classical & $5.619 \pm 0.124$ & $1.520 \pm 0.180$ & & 27 \\
\hline $2000 \mathrm{CQ}_{105}$ & Scattered disk object & $5.996 \pm 0.054$ & $1.107 \pm 0.043$ & & 25,28 \\
\hline $2000 \mathrm{FS}_{53}$ & Cold classical & $7.165 \pm 0.124$ & $1.786 \pm 0.095$ & & 19,27 \\
\hline $2000 \mathrm{FZ}_{53}$ & Centaur & $11.103 \pm 0.165$ & $1.170 \pm 0.050$ & & 25 \\
\hline $2000 \mathrm{KK}_{4}$ & Hot classical & $5.982 \pm 0.103$ & $1.550 \pm 0.050$ & & 19 \\
\hline $2000 \mathrm{PE}_{30}$ & Scattered disk object & $5.867 \pm 0.110$ & $1.132 \pm 0.084$ & & $15,16,21$ \\
\hline $2000 \mathrm{YB}_{2}$ & Scattered disk object & $6.436 \pm 0.084$ & $1.500 \pm 0.134$ & & 31 \\
\hline $2001 \mathrm{FM}_{194}$ & Scattered disk object & $7.453 \pm 0.159$ & $1.190 \pm 0.040$ & & 25 \\
\hline $2001 \mathrm{HY}_{65}$ & Hot classical & $6.041 \pm 0.064$ & $1.510 \pm 0.092$ & & 31 \\
\hline $2001 \mathrm{HZ}_{58}$ & Cold classical & $6.158 \pm 0.053$ & $1.640 \pm 0.085$ & & 31 \\
\hline $2001 \mathrm{KA}_{77}$ & Hot classical & $5.050 \pm 0.089$ & $1.812 \pm 0.122$ & & $8,28,30$ \\
\hline $2001 \mathrm{~KB}_{77}$ & Plutino & $7.349 \pm 0.078$ & $1.390 \pm 0.130$ & & 24 \\
\hline $2001 \mathrm{KD}_{77}$ & Plutino & $5.928 \pm 0.096$ & $1.763 \pm 0.060$ & & 8,28 \\
\hline $2001 \mathrm{KG}_{77}$ & Scattered disk object & $8.340 \pm 0.120$ & $1.240 \pm 0.070$ & & 25 \\
\hline $2001 \mathrm{KY}_{76}$ & Plutino & $6.689 \pm 0.380$ & $1.960 \pm 0.291$ & & 30 \\
\hline $2001 \mathrm{QC}_{298}$ & Hot classical & $6.381 \pm 0.174$ & $1.030 \pm 0.098$ & & 31 \\
\hline $2001 \mathrm{QD}_{298}$ & Hot classical & $6.185 \pm 0.170$ & $1.640 \pm 0.158$ & & 30 \\
\hline $2001 \mathrm{QF}_{298}$ & Plutino & $5.119 \pm 0.118$ & $1.051 \pm 0.085$ & & $15,24,30$ \\
\hline $2001 \mathrm{QR}_{322}$ & Neptune Trojan & $7.828 \pm 0.010$ & $1.260 \pm 0.036$ & & 41 \\
\hline $2001 \mathrm{QX}_{322}$ & Scattered disk object & $6.144 \pm 0.146$ & $1.752 \pm 0.280$ & & 25,31 \\
\hline $2001 \mathrm{QY}_{297}$ & Cold classical & $5.151 \pm 0.231$ & $1.561 \pm 0.177$ & & $15,30,35$ \\
\hline $2001 \mathrm{RZ}_{143}$ & Cold classical & $6.241 \pm 0.123$ & $1.590 \pm 0.191$ & & 31 \\
\hline $2001 \mathrm{XZ}_{255}$ & Centaur & $10.800 \pm 0.080$ & $1.910 \pm 0.070$ & & 25 \\
\hline $2002 \mathrm{DH}_{5}$ & Centaur & $10.115 \pm 0.100$ & $1.054 \pm 0.075$ & & 28 \\
\hline $2002 \mathrm{~GB}_{32}$ & Scattered disk object & $7.638 \pm 0.019$ & $1.390 \pm 0.020$ & & 21 \\
\hline $2002 \mathrm{GF}_{32}$ & Plutino & $5.973 \pm 0.210$ & $1.765 \pm 0.134$ & & 30 \\
\hline $2002 \mathrm{GH}_{32}$ & Hot classical & $6.098 \pm 0.201$ & $1.509 \pm 0.160$ & & 30,31 \\
\hline $2002 \mathrm{GP}_{32}$ & Resonant (5:2) & $6.580 \pm 0.162$ & $1.386 \pm 0.162$ & & 30,35 \\
\hline $2002 \mathrm{GV}_{32}$ & Plutino & $6.886 \pm 0.199$ & $1.860 \pm 0.122$ & & 30 \\
\hline $2002 \mathrm{MS}_{4}$ & Resonant (18:11) & $3.333 \pm 0.040$ & $1.070 \pm 0.040$ & & 24 \\
\hline $2002 \mathrm{VT}_{130}$ & Cold classical & $5.426 \pm 0.092$ & $2.010 \pm 0.233$ & & 31 \\
\hline $2002 \mathrm{XV}_{93}$ & Plutino & $4.434 \pm 0.040$ & $1.090 \pm 0.030$ & & 24 \\
\hline $2003 \mathrm{AZ}_{84}$ & Plutino & $3.537 \pm 0.053$ & $1.052 \pm 0.057$ & Methanol & $24,31,33, \mathrm{~h}$ \\
\hline $2003 \mathrm{FZ}_{129}$ & Scattered or detached KBO & $6.983 \pm 0.038$ & $1.320 \pm 0.040$ & & 21 \\
\hline $2003 \mathrm{HB}_{57}$ & Scattered or detached KBO & $7.389 \pm 0.028$ & $1.310 \pm 0.030$ & & 21 \\
\hline $2003 \mathrm{QA}_{92}$ & Scattered disk object & $6.367 \pm 0.240$ & $1.670 \pm 0.020$ & & 37 \\
\hline $2003 \mathrm{QK}_{91}$ & Scattered or detached KBO & $6.966 \pm 0.036$ & $1.370 \pm 0.040$ & & 21 \\
\hline $2003 \mathrm{QQ}_{91}$ & Scattered disk object & $7.624 \pm 0.280$ & $1.180 \pm 0.050$ & & 37 \\
\hline $2003 \mathrm{QW}_{90}$ & Hot classical & $4.730 \pm 0.057$ & $1.780 \pm 0.092$ & & 31 \\
\hline $2003 \mathrm{TH}_{58}$ & Plutino & $6.940 \pm 0.056$ & $0.990 \pm 0.071$ & & 40 \\
\hline $2003 \mathrm{UZ}_{117}$ & Hot classical & $4.920 \pm 0.083$ & $0.990 \pm 0.050$ & Water ice & $24, \mathrm{q}$ \\
\hline 2003 YL $_{179}$ & Cold classical & $7.482 \pm 0.300$ & $1.260 \pm 0.090$ & & 37 \\
\hline $2004 \mathrm{OJ}_{14}$ & Scattered or detached KBO & $6.991 \pm 0.028$ & $1.420 \pm 0.030$ & & 21 \\
\hline $2004 \mathrm{UP}_{10}$ & Neptune Trojan & $8.651 \pm 0.030$ & $1.160 \pm 0.064$ & & 41 \\
\hline $2004 X_{190}$ & Detached KBO & $3.937 \pm 0.036$ & $1.240 \pm 0.040$ & & 21 \\
\hline $2005 \mathrm{CB}_{79}$ & Hot classical & $4.375 \pm 0.028$ & $1.090 \pm 0.028$ & Water ice & $40, \mathrm{q}$ \\
\hline $2005 \mathrm{EO}_{297}$ & Resonant $(3: 1)$ & $7.221 \pm 0.047$ & $1.320 \pm 0.050$ & & 21 \\
\hline $2005 \mathrm{GE}_{187}$ & Plutino & $7.192 \pm 0.097$ & $1.740 \pm 0.112$ & & 40 \\
\hline $2005 \mathrm{PU}_{21}$ & Scattered disk object & $6.091 \pm 0.019$ & $1.790 \pm 0.020$ & & 21 \\
\hline $2005 \mathrm{SD}_{278}$ & Scattered or detached KBO & $5.915 \pm 0.019$ & $1.530 \pm 0.020$ & & 21 \\
\hline $2005 \mathrm{TN}_{53}$ & Neptune Trojan & $9.027 \pm 0.040$ & $1.290 \pm 0.106$ & & 41 \\
\hline $2005 \mathrm{TO}_{74}$ & Neptune Trojan & $8.426 \pm 0.030$ & $1.340 \pm 0.078$ & & 41 \\
\hline $2006 \mathrm{RJ}_{103}$ & Neptune Trojan & $7.400 \pm 0.023$ & $1.903 \pm 0.044$ & & This work \\
\hline $2006 \mathrm{SQ}_{372}$ & Scattered disk object & $7.709 \pm 0.049$ & $1.712 \pm 0.093$ & & 21, This work \\
\hline $2007 \mathrm{JJ}_{43}$ & Hot classical & $4.044 \pm 0.019$ & $1.610 \pm 0.020$ & & 21 \\
\hline $2007 \mathrm{JK}_{43}$ & Scattered disk object & $7.028 \pm 0.017$ & $1.400 \pm 0.027$ & & This work \\
\hline $2007 \mathrm{NC}_{7}$ & Scattered disk object & $8.068 \pm 0.018$ & $1.282 \pm 0.028$ & & This work \\
\hline $2007 \mathrm{RH}_{283}$ & Centaur & $8.435 \pm 0.039$ & $1.237 \pm 0.069$ & & This work \\
\hline $2007 \mathrm{TG}_{422}$ & Scattered disk object & $6.186 \pm 0.010$ & $1.390 \pm 0.040$ & & 21 \\
\hline $2007 \mathrm{UM}_{126}$ & Centaur & $10.161 \pm 0.042$ & $1.080 \pm 0.096$ & Water ice & This work, i \\
\hline $2007 \mathrm{VJ}_{305}$ & Scattered disk object & $6.713 \pm 0.028$ & $1.440 \pm 0.030$ & & 21 \\
\hline $2008 \mathrm{FC}_{76}$ & Centaur & $9.181 \pm 0.039$ & $1.756 \pm 0.024$ & Methanol & This work, i \\
\hline $2008 \mathrm{KV}_{42}$ & Scattered disk object & $8.564 \pm 0.056$ & $1.290 \pm 0.060$ & & 21 \\
\hline $2008 \mathrm{OG}_{19}$ & Scattered or detached KBO & $4.612 \pm 0.013$ & $1.470 \pm 0.010$ & & 21 \\
\hline
\end{tabular}

References. see Appendix A. 


\section{Appendix A: Compiled database}

For each object, we computed the average color index from the different papers presenting data obtained simultaneously in $B$ and $R$ bands (e.g. contiguous observations within a same night). When individual $R$ apparent magnitude and date were available, we computed the $H_{R}(\alpha)=R-5 \log (r \cdot \Delta)$, where $R$ is the $R$-band magnitude, $r$ and $\Delta$ are the helio- and geocentric distances at the time of observation in AU, respectively. When $V$ and $V-R$ colors were available, we derived an $R$ and then $H_{R}(\alpha)$ value. We did not correct for the phase-angle $\alpha$ effect as we needed only to make a general estimation of the absolute magnitude for our complete sample. In addition, few objects have phase correction coefficients available in the literature, and no universally accepted canonical values per dynamical class can be strictly adopted. Table 3 presents the resulting values. This table includes also spectral information on the presence of water ice, methanol, methane, or confirmed featureless spectra, as available in the literature. We highlight only the cases with clear bands in the spectrum, which were reported/confirmed by some other work.

There is no strict definition of the dynamical classes of Centaurs and KBOs. Roughly speaking, objects orbiting in mean motion resonances with Neptune are called "resonants" (if located in the 1:1 resonance they are also known as Neptune Trojans and as Plutinos if located in the 3:2 resonance); Centaurs are the objects with orbits between those of Jupiter and Neptune; scattered disk objects (SDOs), are those within the probable gravitational influence of Neptune; detached KBOs, are those beyond past or future gravitational influence by Neptune; classical KBOs, are those with rather circular orbits beyond Neptune and below the 2:1 resonance region (being called hot if their orbital inclination is higher than $5^{\circ}$ or cold if lower).

To determine the dynamical class, we first gathered the orbital elements, with epoch 2011-12-05, from "The Asteroid Orbital Elements Database", astorb.dat ${ }^{1}$, maintained by the "Lowell Observatory" based on astrometric observations by the "Minor Planet Center". Then, using the particular classification scheme suggested by Lykawka \& Mukai (2007), including their analysis of objects located in the mean motion resonances (MMR) with Neptune, dynamical class was determined following a 11 step algorithm:

1. $q<a_{\mathrm{J}} \Rightarrow$ Not analyzed;

2. in 1:1 MMR with Neptune $\Rightarrow$ Neptune Trojan;

3. in 3:2 MMR with Neptune $\Rightarrow$ Plutino;

4. in other MMR with Neptune $\Rightarrow$ other resonant;

5. $q>a_{\mathrm{J}} \wedge a<a_{\mathrm{N}} \Rightarrow$ Centaur;

6. $a_{\mathrm{J}}<q<a_{\mathrm{N}} \wedge a \geqslant a_{\mathrm{N}} \Rightarrow$ scattered disk object (SDO);
7. $a_{\mathrm{N}}<q \leqslant 37 \mathrm{AU} \Rightarrow$ scattered disk object (SDO);

8. $q \geqslant 40 \mathrm{AU} \wedge a \geqslant 48 \mathrm{AU} \Rightarrow$ detached KBO (DKBO);

9. $37 \mathrm{AU} \leqslant q \leqslant 40 \mathrm{AU} \Rightarrow$ scattered or detached $\mathrm{KBO}$ (SDKBO);

10. $i<5^{\circ} \wedge\{[q \geqslant 37 \mathrm{AU} \wedge(37 \mathrm{AU} \leqslant a \leqslant 40 \mathrm{AU})] \vee[q \geqslant$ $38 \mathrm{AU} \wedge(42 \mathrm{AU} \leqslant a \leqslant 48 \mathrm{AU})]\} \Rightarrow$ cold classical KBO $(\mathrm{cCKBO})$

11. $i \geqslant 5^{\circ} \wedge q \geqslant 37 \mathrm{AU} \wedge(37 \mathrm{AU} \leqslant a \leqslant 48 \mathrm{AU}) \Rightarrow$ hot classical $\mathrm{KBO}(\mathrm{hCKBO})$.

where $q$ and $a$ are the object's perihelion and semi-major axis, respectively. Jupiter semi-major axis is $a_{\mathrm{J}}$, and Neptune's is $a_{\mathrm{N}}$. We note that throughout the algorithm an object can be reclassified.

We are aware that there are more complex classification schemes, which may be more refined, but the boundaries between families do not change significantly. We chose this one for its computational simplicity.

References for the colors presented in Table 3 are: (1) Luu \& Jewitt (1996); (2) Lazzaro et al. (1997); (3) Romon-Martin et al. (2003); (4) Romanishin et al. (1997); (5) Romon-Martin et al. (2002); (6) Tegler \& Romanishin (1998); (7) Jewitt \& Luu (2001); (8) Doressoundiram et al. (2002); (9) Tegler \& Romanishin (2000); (10) Delsanti et al. (2001); (11) Tegler \& Romanishin (1997); (12) Jewitt \& Luu (1998); (13) Barucci et al. (1999); (14) Boehnhardt et al. (2001); (15) Doressoundiram et al. (2007); (16) Doressoundiram et al. (2001); (17) Green et al. (1997); (18) Boehnhardt et al. (2002); (19) Tegler \& Romanishin (2003); (20) Hainaut et al. (2000); (21) Sheppard (2010); (22) Barucci et al. (2000); (23) Rabinowitz et al. (2008); (24) Tegler et al. ${ }^{2}$; (25) Tegler et al. (2003); (26) Peixinho et al. (2001); (27) Trujillo \& Brown (2002); (28) Peixinho et al. (2004); (29) Ferrin et al. (2001); (30) Doressoundiram et al. (2005b); (31) Santos-Sanz et al. (2009); (32) Dotto et al. (2003); (33) Fornasier et al. (2004); (34) Doressoundiram et al. (2005a); (35) Gulbis et al. (2006); (36) Rabinowitz et al. (2007); (37) Romanishin et al. (2010); (38) Rabinowitz et al. (2006); (39) Lacerda et al. (2008); (40) Snodgrass et al. (2010); (41) Sheppard \& Trujillo (2006).

References for the spectral features indicated in Table 3 are: (a) Romon-Martin et al. (2003); (b) Cruikshank et al. (1998); (c) Kern et al. (2000); (d) Guilbert et al. (2009b); (e) Jewitt \& Luu (2001); (f) Brown et al. (1999); (g) Barkume et al. (2008); (h) Guilbert et al. (2009a); (i) Barucci et al. (2011); (j) DeMeo et al. (2010); (k) Grundy et al. (2005); (1) Barucci et al. (2010); (m) Delsanti et al. (2010); (n) Pinilla-Alonso et al. (2009); (o) Merlin et al. (2009); (p) Brown et al. (2007a); (q) Schaller \& Brown (2008).

\footnotetext{
1 ftp://ftp.lowell.edu/pub/elgb/astorb.dat.gz
}

2 http://www.physics.nau.edu/ tegler/research/survey. htm 